\title{
A Guided Walk Down Wall Street: An Introduction to Econophysics
}

\author{
Giovani L. Vasconcelos \\ Laboratório de Física Teórica e Computacional, Departamento de Física, \\ Universidade Federal de Pernambuco, 50670-901 Recife, PE, Brazil
}

Received on 10 May, 2004

\begin{abstract}
This article contains the lecture notes for the short course "Introduction to Econophysics," delivered at the II Brazilian School on Statistical Mechanics, held in São Carlos, Brazil, in February 2004. The main goal of the present notes is twofold: i) to provide a brief introduction to the problem of pricing financial derivatives in continuous time; and ii) to review some of the related problems to which physicists have made relevant contributions in recent years.
\end{abstract}

\section{Introduction}

This article comprises the set of notes for the short course "Introduction to Econophysics," delivered at the II Brazilian School on Statistical Mechanics, held at the University of São Paulo, in São Carlos, SP, Brazil, in February 2004. The course consisted of five lectures and was aimed at physics graduate students with no previous exposition to the subject.

The main goal of the course was twofold: i) to provide a brief introduction to the basic models for pricing financial derivatives; and ii) to review some of the related problems in Finance to which physicists have made significant contributions over the last decade. The recent body of work done by physicists and others have produced convincing evidences that the standard model of Finance (see below) is not fully capable of describing real markets, and hence new ideas and models are called for, some of which have come straight from Physics. In selecting some of the more recent work done by physicists to discuss here, I have tried to restrict myself to problems that may have a direct bear on models for pricing derivatives. And even in such cases only a brief overview of the problems is given. It should then be emphasized that these notes are not intended as a review article on Econophysics, which is nowadays a broad interdisciplinary area, but rather as a pedagogical introduction to the mathematics (and physics?) of financial derivatives. Hence no attempt has been made to provide a comprehensive list of references.

No claim of originality is made here regarding the contents of the present notes. Indeed, the basic theory of financial derivatives can now be found in numerous textbooks, written at a different mathematical levels and aiming at specific (or mixed) audiences, such as economists [1, 2, 3, 4], applied mathematicians $[5,6,7,8]$, physicists $[9,10,11]$, etc. (Here I have listed only the texts that were most often consulted while writing these notes.) Nevertheless, some aspects of presentation given here have not, to my knowledge, appeared before. An example is the analogy between market efficiency and a certain symmetry principle that is put forward in Sec. V. Similarly, the discussion of some of the more recent research problems is based on the already published literature. An exception is Fig. 12 which contains unpublished results obtained by R. L. Costa and myself.

The present notes are organized as follows. Section II gives some basic notions of Finance, intended to introduce the terminology as well as the main problems that I shall be considering. In Sec. III, I discuss the Brownian motion, under a more formal viewpoint than most Physics graduate students are perhaps familiar with, and then develop the so-called Itô stochastic calculus. Section IV contains what is the raison d'etre of the present notes, the Black-Scholes model for pricing financial derivatives. In Sec. V, the martingale approach for pricing derivatives is introduced. In particular, I recast the notions of market efficiency and no-arbitrage as a 'symmetry principle' and its associated 'conservation law.' Sections VI and VII discuss two possible ways in which real markets may deviate from the standard Black-Scholes model. The first of such possibilities is that financial asset prices have non-Gaussian distributions (Sec. VI), while the second one concerns the presence of long-range correlations or memory effects in financial data (Sec. VII). Conclusions are presented in Sec. VIII. For completeness, I give in Appendix A the formal definitions of probability space, random variables, and stochastic processes.

\section{Basic Notions of Finance}

\subsection{Riskless and risky financial assets}

Suppose you deposit at time $t=0$ an amount of $\mathrm{R} \$ 1$ into a bank account that pays an interest rate $r$. Then over time the amount of money you have in the bank, let us call it $B(t)$, will increase at a rate

$$
\frac{d B}{d t}=r B
$$

Solving this equation subject to the initial condition $B(0)=$ 1 yields

$$
B(t)=e^{r t} .
$$


A bank account is an example of a riskless financial assets, since you are guaranteed to receive a known (usually fixed) interest rate $r$, regardless of the market situation. Roughly speaking, the way banks operate is that they borrow from people who have money to 'spare', but are not willing to take risks, and lend (at higher interest rates) to people who 'need' money, say, to invest in some risky enterprise. By diversifying their lending, banks can reduce their overall risk, so that even if some of these loans turn bad they can still meet their obligations to the investors from whom they borrowed.

Governments and private companies can also borrow money from investors by issuing bonds. Like a bank account, a bond pays a (fixed or floating) interest rate on a regular basis, the main difference being that the repayment of the loan occurs only at a specified time, called the bond maturity. Another difference is that bonds are not strictly riskfree assets because there is always a chance that the bond issuer may default on interest payments or (worse) on the principal. However, since governments have a much lower risk to default than corporations, certain government bonds can be considered to be risk free.

A company can also raise capital by issuing stocks or shares. Basically, a stock represents the ownership of a small piece of the company. By selling many such 'small pieces', a company can raise capital at lower costs than if it were to borrow from a bank. As will be discussed shortly, stocks are risky financial assets because their prices are subjected to unpredictable fluctuations. In fact, this is what makes stocks attractive to aggressive investors who seek to profit from the price fluctuations by pursuing the old advice to "buy low and sell high."

The buying and selling of stocks are usually done in organized exchanges, such as, the New York Stock Exchange (NYSE) and the São Paulo Stock Exchange (BOVESPA). Most stock exchanges have indexes that represent some sort of average behavior of the corresponding market. Each index has its own methodology. For example, the Dow Jones Industrial Average of the NYSE, which is arguably the most famous stock index, corresponds to an average over 30 industrial companies. The Ibovespa index of the São Paulo Stock Exchange, in contrast, represents the present value of a hypothetical portfolio made up of the stocks that altogether correspond to $80 \%$ of the trading volume. Another well known stock index is the Standard \& Poor's 500 (S\&P500) Index calculated on the basis of data about 500 companies listed on the NYSE. [Many other risky financial assets, such as, currency exchange rates, interest rates, and commodities (precious metals, oil, grains, etc), are traded on organized markets but these will not be discussed any further in the present notes.]

\subsection{The random nature of stock prices}

Since a stock represents a 'small piece' of a company, the stock price should somehow reflect the overall value (net worth) of this company. However, the present value of a firm depends not only on the firm's current situation but also on its future performance. So here one sees already the basic problem in pricing risky financial assets: we are trying to predict the future on the basis of present information. Thus, if a new information is revealed that might in one way or another affect the company's future performance, then the stock price will vary accordingly. It should therefore be clear from this simple discussion that the future price of a stock will always be subjected to a certain degree of uncertainty. This is reflected in the typical 'erratic behavior' that stock prices show when graphed as a function of time. An example of such a graph is shown in Fig. 1 for the case of the Ibovespa stock index.

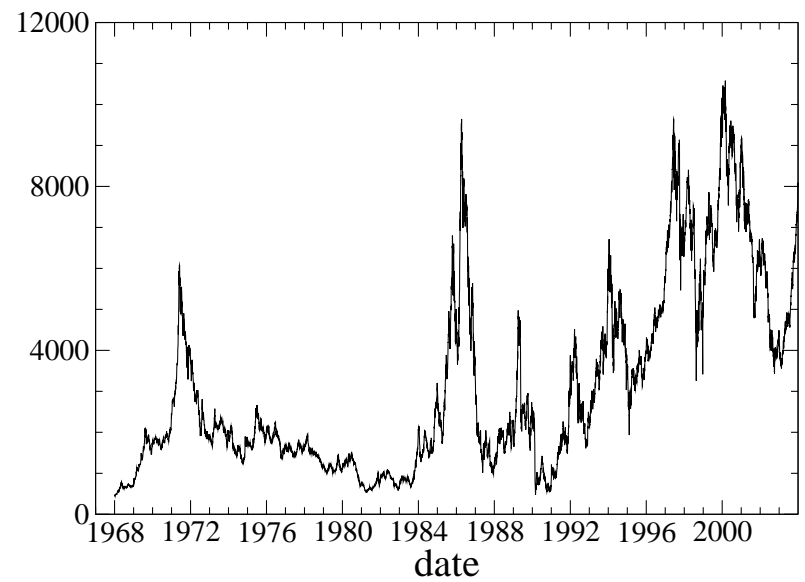

Figure 1. Daily closing values of the deflated Ibovespa index in the period 1968-2003.

Although stock prices may vary in a rather unpredictable way, this does not mean that they cannot be modeled. It says only that they should be described in a probabilistic fashion. To make the argument a little more precise, let $S$ be the price of a given stock and suppose we want to write an equation analogous to (1) for the rate of increase of $S$ :

$$
\frac{d S}{d t}=R(t) S
$$

where $R(t)$ represents the 'rate of return' of the stock. The question then is: what is $R(t)$ ? From our previous discussion, it is reasonable to expect that $R(t)$ could be separated into two components: i) a predictable mean rate of return, to be denoted by $\mu$, and ii) a fluctuating ('noisy') term $\xi(t)$, responsible for the randomness or uncertainty in the stock price. Thus, after writing $R(t)=\mu+\xi(t)$ in (3) we have

$$
\frac{d S}{d t}=[\mu+\xi(t)] S
$$

Now, one of the best models for 'noise' is, of course, the white noise, so it should not come as a surprise to a physicist that Brownian motion and white noise play an important rôle in finance, as will be discussed in detail shortly.

\subsection{Options and derivatives}

Besides the primary financial assets already mentioned (stocks, commodities, exchange rate, etc), many other financial instruments, such as options and futures contracts, 
are traded on organized markets (exchanges). These securities are generically called derivatives, because they derive their value from the price of some primary underlying asset. Derivatives are also sometimes referred to as contingent claims, since their values are contingent on the evolution of the underlying asset. In the present notes, I will discuss only one of the most basic derivatives, namely, options.

An option is a contract that gives its holder the right, but not the obligation, to buy or sell a certain asset for a specified price at some future time. The other part of the contract, the option underwriter, is obliged to sell or buy the asset at the specified price. The right to buy (sell) is called a call (put) option. If the option can only be exercised at the future date specified in the contract, then it is said to be a European option. American options, on the other hand, can be exercised at any time up to maturity. (For pedagogical reasons, only European derivatives will be considered here.) To establish some notation let us give a formal definition of a European option.

Definition 1 A European call option with exercise price (or strike price) $K$ and maturity (or expiration date) $T$ on the underlying asset $S$ is a contract that gives the holder the right to buy the underlying asset for the price $K$ at time $T$.

A European put option is the same as above, the only difference being that it gives the holder the right to sell the underlying asset for the exercise price at the expiration date.

If at the expiration date $T$ the stock price $S_{T}$ is above the strike price $K$, the holder of a call option will exercise his right to buy the stock from the underwriter at price $K$ and sell it in the market at the spot price $S_{T}$, pocketing the difference $S_{T}-K$. On the other hand, if at expiration the price $S_{T}$ closes below $K$ then the call option becomes worthless (since it would be cheaper to buy the stock in the market). The payoff of a call option at maturity is therefore given by

$$
\text { payoff }_{\text {call }}=\max \left(S_{T}-K, 0\right) \text {. }
$$

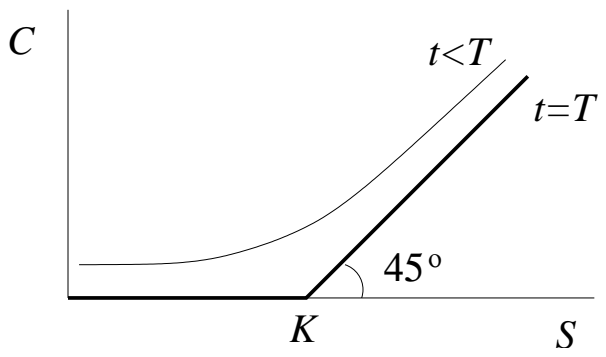

Figure 2. Value of a call option at the expiration date (thick line) and before expiration (thin line).

The payoff diagram of a call option is illustrated by the thick line in Fig. 2. In this figure the thin line represents the price of the call option at an arbitrary time $t<T$ before expiration. (The otpion price before expiration is always greater than the payoff at expiration on account of the higher risks: the further way the expiration date, the greater the uncertainty regarding the stock price at expiration.) Similarly, the payoff function for a put option is

$$
\text { payoff }_{\text {put }}=\max \left(K-S_{T}, 0\right),
$$

which is shown as the thick line in Fig. 3.

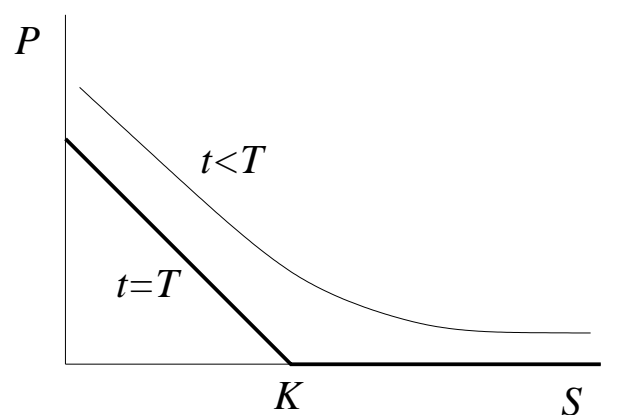

Figure 3. Value of a put option at the expiration date (thick line) and before expiration (thin line).

Because an option entitles the holder to a certain right it commands a premium. Said differently, since the underwriter has an obligation (while the holder has only rights) he will demand a payment, to be denoted by $C_{0}$, from the holder in order to enter into such a contract. Thus, in the case of a call option, if the option is exercised the holder (underwriter) will make a profit (loss) given by $\max (S-$ $K, 0)-C_{0}$; otherwise, the holder (underwriter) will have lost (won) the amount $C_{0}$ paid (received) for the option. And similarly for a put option. Note then that the holder and the underwriter of an option have opposite views regarding the direction of the market. For instance, the holder of a call option is betting that the stock price will increase (past the exercise price), whereas the underwriter hopes for the opposite.

Now, given that the holder and the underwriter have opposite views as to the direction of the market, how can they possibly agree on the price for the option? For if the holder (underwriter) suspects that the option is overvalued (undervalued) he will walk away from the contract. The central problem in option pricing is therefore to determine the $\mathrm{ra}$ tional price price $C_{0}$ that ensures that neither part 'stands a better chance to win.'

A solution to this problem (under certain assumptions) was given in 1973 in the now-famous papers by Black and Scholes [12] and Merton [13], which won Scholes and Merton the Nobel prize in Economics in 1997. (Black had died meanwhile.) The history of options is however much longer. In fact, the first scientific study of options dates back to the work by the French mathematician Bachelier in 1900 [14], who solved the option pricing problem above but under slightly wrong assumptions; see, e.g., [11] for a detailed discussion of Bachelier's work.

After an option (traded on exchange) is first underwritten, it can subsequently be traded and hence its 'market price' will be determined by the usual bid-ask auction. It is nonetheless important to realize that investors in such highly specialized market need some basic pricing theory to rely on, otherwise investing in options would be a rather wild (and dangerous) game. Indeed, only after the appearance 
of the Black-Scholes model [and the establishment of the first option exchange in Chicago also in 1973] have option markets thrived. One of the main objectives of the present notes is to explain the theoretical framework, namely, the Black-Scholes model and some of its extensions, in which options and other derivatives are priced. I will therefore not say much about the practical aspects of trading with options.

\subsection{Hedging, speculation, and arbitrage}

Investors in derivative markets can be classified into three main categories: hedgers, speculators, and arbitrageurs.

Hedgers are interested in using derivatives to reduce the risk they already face in their portfolio. For example, suppose you own a stock and are afraid that its price might go down within the next months. One possible way to limit your risk is to sell the stock now and put the money in a bank account. But then you won't profit if the market goes up. A better hedging strategy would clearly be to buy a put option on the stock, so that you only have to sell the stock if it goes below a certain price, while getting to keep it if the price goes up. In this case an option works pretty much as an insurance: you pay a small price (the option premium $C_{0}$ ) to insure your holdings against possibly high losses.

Speculators, in contrast to hedgers, seek to make profit by taking risks. They 'take a position' in the market, by betting that the price on a given financial asset will go either up or down. For instance, if you think that a certain stock will go up in the near future, you could "buy and hold" the stock in the hope of selling it later at a profit. But then there is the risk that the price goes down. A better strategy would thus be to buy a call option on the stock. This not only is far cheaper than buying the stock itself but also can yield a much higher return on your initial investment. (Why?) However, if the market does not move in the way you expected and the option expire worthless, you end up with a $100 \%$ loss. (That's why speculating with option is a very risky business.)

Arbitrageurs seek to make a riskless profit by entering simultaneously into transactions in two or more markets, usually without having to make any initial commitment of money. The possibility of making a riskless profit, starting with no money at all, is called an arbitrage opportunity or, simply, an arbitrage. A more formal definition of arbitrage will be given later. For the time being, it suffices to give an example of how an arbitrage opportunity may arise.

But before going into this example, it is necessary first to discuss the notion of a short sell. 'Shorting' means selling an asset that one does not own. For example, if you place an order to your broker to short a stock, the broker will "borrow" a stock from somebody else's account, sell it in the market, and credit the proceeds into your account. When you then decide to close your short position (there usually is a limit on how long an asset can be held short), your broker will buy the stock in the market (taking the money from your account) and return it to its original owner. If in the meantime the stock prices decreased, the short sell brings a profit, otherwise the short seller incurs in a loss. This is why a short sell is usually done simultaneously with another oper- ation to compensate for this risk (as in the arbitrage example below). It should also be noted, in passing, that buying the actual asset corresponds to taking a 'long position' on this asset.

Let us now consider our hypothetical arbitrage example. Many Brazilian companies listed in the São Paulo Stock Exchange also have their stocks traded on the New York Stock Exchange in the form of the so-called American Depository Receipt (ADR). Suppose then that a stock is quoted in São Paulo at R\$100, with its ADR counterpart trading in New York at US\$ 34, while the currency rate exchange is $1 \mathrm{USD}=2.90$ BRL. Starting with no initial commitment, an arbitrageur could sell short $N$ stocks in São Paulo and use the proceeds to buy $N$ ADR's in New York (and later have them transferred to São Paulo to close his short position). The riskless profit in such operation would be $\mathrm{R} \$$ $(100-2.90 \times 34) N=\mathrm{R} \$ 1.40 N$. (In practice, the transaction costs would eliminate the profit for all but large institutional investors [1].)

Note, however, that such 'mispricing' cannot last long: buy orders in New York will force the ADR price up, while sell orders in São Paulo will have the opposite effect on the stock price, so that an equilibrium price for both the ADR and the stock is soon reached, whereupon arbitrage will no longer be possible. In this sense, the actions of an arbitrageur are self-defeating, for they tend to destroy the very arbitrage opportunity he is acting upon-but before this happens a lot of money can be made. Since there are many people looking for such riskless chances to make money, a well-functioning market should be free of arbitrage. This is the main idea behind the principle that in an efficient market there is no arbitrage, which is commonly known as the "no-free-lunch" condition.

\subsection{The no-arbitrage principle in a (binomial) nutshell}

Here we shall consider a one-step binomial model to illustrate the principle of no-arbitrage and how it can be used to price derivatives. Suppose that today's price of an ordinary Petrobras stocks (PETR3 in their Bovespa acronym) is $S_{0}=57$ BRL. Imagine then that in the next time-period, say, one month, the stock can either go up to $S_{1}^{u}=65$ with probability $p$ or go down to $S_{1}^{d}=53$ with probability $q$. For simplicity let us take $p=q=1 / 2$. Our binomial model for the stock price dynamics is illustrated in Fig. 4. Note that in this case the stock mean rate of return, $\mu$, is given by the expression: $(1+\mu) S_{0}=E\left[S_{1}\right]$, where $E[S]$ denotes the expected value of $S$ (see Sec. III A for more on this notation). Using the values shown in Fig. 4, one then gets $\mu=0.035$ or $\mu=3.5 \%$. Let us also assume that the risk-free interest rate is $r=0.6 \%$ monthly.

Consider next a call option on PETR3 with exercise price $K=57$ and expiration in the next time period, i.e., $T=1$. Referring to (5) and Fig. 4, one immediately sees that at expiration the possible values (payoffs) for this option in our binomial model are as shown in Fig. 5: $C_{1}^{u}=8$ or $C_{1}^{d}=0$ with equal probability. The question then is to determine the 'rational' price $C_{0}$ that one should pay for the 
option. Below we will solve this problem using two different but related methods. The idea here is to illustrate the main principles involved in option pricing, which will be generalized later for the case of continuous time.

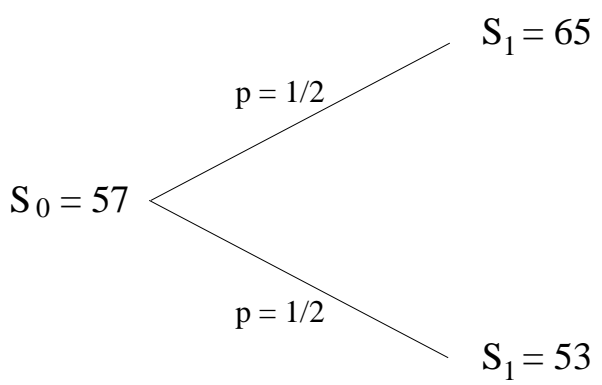

Figure 4. One-step binomial model for a stock.

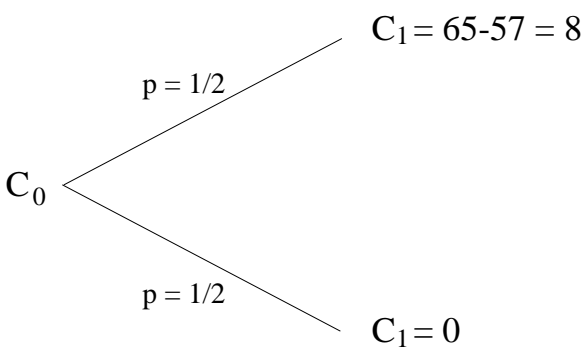

Figure 5. Option value in the one-step binomial model.

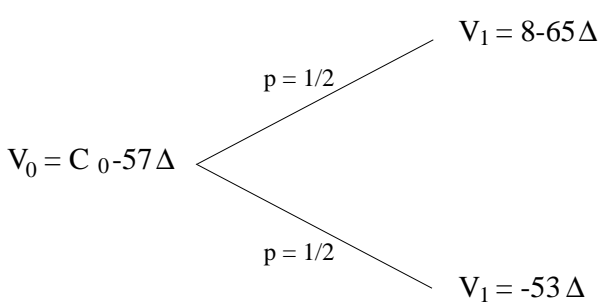

Figure 6. Delta-hedging portfolio in the one-step binomial model.

First, we describe the so-called delta-hedging argument. Consider a portfolio made up of one option $C$ and a short position on $\Delta$ stocks, where $\Delta$ is to be determined later, and let $V_{t}$ denote the money value of such a portfolio at time $t$. We thus have

$$
V_{t}=C_{t}-\Delta S_{t},
$$

where the minus sign denotes that we have short sold $\Delta$ stocks (i.e., we 'owe' $\Delta$ stocks in the market). From Figs. 4 and 5 , one clearly sees that the possibles values for this portfolio in our one-step model are as illustrated in Fig. 6. Let us now chose $\Delta$ such that the value $V_{1}$ of the portfolio is the same in both 'market situations.' Referring to Fig. 6 one immediately finds

$$
V_{1}^{u}=V_{1}^{d} \Longrightarrow 8-\Delta \cdot 65=-\Delta \cdot 53 \Longrightarrow \Delta=\frac{2}{3} .
$$

Thus, by choosing $\Delta=2 / 3$ we have completely eliminated the risk from our portfolio, since in both (up or down) scenarios the portfolio has the same value $V_{1}$. But since this portfolio is riskless, its rate of return must be equal to the risk-free interest rate $r$, otherwise there would be an arbitrage opportunity, as the following argument shows.

Let $r^{\prime}$ denote the portfolio rate of return, i.e., $r^{\prime}$ is the solution to the following equation

$$
\left(1+r^{\prime}\right) V_{0}=V_{1} \text {. }
$$

If $r^{\prime}<r$, then an arbitrageur should take a long position on (i.e., buy) the option and a short position on $\Delta$ stocks. To see why this is an arbitrage, let us go through the argument in detail. At time $t=0$ the arbitrageur's net cashflow would be $B_{0}=\left|V_{0}\right|=\Delta \cdot S_{0}-C_{0}$, which he should put in the bank so that in the next period he would have $B_{1}=(1+r)\left|V_{0}\right|$. At time $t=1$, he should then close his short position on $\Delta$ stocks, either exercising his option (up scenario) or buying $\Delta$ stocks directly in the market (down scenario). In either case, he would have to pay the same amount $\left|V_{1}\right|=\left(1+r^{\prime}\right)\left|V_{0}\right|<B_{1}$, and hence would be left with a profit of $B_{1}-\left|V_{1}\right|$. On the other hand, if $r^{\prime}>r$ the arbitrageur should adopt the opposite strategy: go short on (i.e., underwrite) the option and long on $\Delta$ stocks (borrowing money from the bank to do so).

We have thus shown that to avoid arbitrage we must have $r^{\prime}=r$. This is indeed a very general principle that deserves to be stated in full: in a market free of arbitrage any riskless portfolio must yield the risk-free interest rate $r$. This no-arbitrage principle is at the core of the modern theory of pricing derivatives, and, as such, it will be used several times in these notes.

Let us now return to our option pricing problem. Setting $r^{\prime}=r$ in (7) and substituting the values of $V_{0}$ and $V_{1}$ given in Fig. 6, we obtain

$$
(1+r)\left[C_{0}-\Delta S_{0}\right]=-\Delta S_{1}^{d}
$$

Inserting the values of $r=0.006, S_{0}=57, S_{1}^{d}=53$, and $\Delta=2 / 3$ into the equation above, it then follows that the option price that rules out arbitrage is

$$
C_{0}=2.88 \text {. }
$$

It is instructive to derive the option price through a second method, namely, the martingale approach or riskneutral valuation. To this end, we first note that from Fig. 5 we see that the expected value of the option at expiration is $E\left[C_{1}\right]=\frac{1}{2} 8+\frac{1}{2} 0=4$. One could then think, not totally unreasonably, that the correct option price should be the expected payoff discounted to the present time with the risk-free interest rate. In this case one would get

$$
C_{0}^{\prime}=\frac{E\left[C_{1}\right]}{1+r}=\frac{4}{1.006}=3.98,
$$

which is quite different from the price found in (9). The faulty point of the argument above is that, while we used the risk-free rate $r$ to discount the expected payoff $E\left[C_{1}\right]$, we have implicitly used the stock mean rate of return $\mu$ when calculating $E\left[C_{1}\right]$. Using these two different rates leads to a wrong price, which would in turn give rise to an arbitrage opportunity. 
A way to avoid this arbitrage is to find fictitious probabilities $q_{u}$ and $q_{d}$, with $q_{u}+q_{d}=1$, such that the stock expected return calculated with these new probabilities would equal the risk-free rate $r$. That is, we must demand that

$$
S_{0}(1+r)=E^{Q}\left[S_{1}\right] \equiv q_{u} \cdot S_{1}^{\mathrm{u}}+q_{d} \cdot S_{1}^{\mathrm{d}},
$$

where $E^{Q}[x]$ denotes expected value with respect to the new probabilities $q_{u}$ and $q_{d}$. Using the values for $S_{1}^{\mathrm{u}}$ and $S_{1}^{\mathrm{d}}$ given in Fig. 4, we easily find that

$$
q_{u}=0.3618, \quad q_{d}=0.6382 .
$$

Under these probabilities, the expected value $E^{Q}\left[C_{1}\right]$ of the option at maturity becomes $E^{Q}\left[C_{1}\right]=0.3618 \times 8+0.6382 \times$ $0=2.894$, which discounted to the present time yields

$$
C_{0}=\frac{E^{Q}\left[C_{1}\right]}{1+r}=\frac{2.894}{1.006}=2.88,
$$

thus recovering the same price found with the delta-hedging argument.

Note that under the fictitious probability $q_{u}$ and $q_{d}$, all financial assets (bank account, stock, and option) in our binomial model yield exactly the same riskless rate $r$. Probabilities that have this property of 'transforming' risky assets into seemingly risk-free ones are called an equivalent martingale measure. Martingale measures is a topic of great relevance in Finance, as will be discussed in more detail in Sec. IV.

In closing this subsection, it should be noted that the one-step binomial model considered above can be easily generalized to a binomial tree with, say, $N$ time steps. But for want of space this will not be done here. (I anticipare here, however, that Black-Scholes model to be considered later corresponds precisely to the continuous-time limit of the binomial multistep model.) It is perhaps also worth mentioning that binomial models are often used in practice to price exotic derivatives, for which no closed formula exists, since such models are rather easy to implement on the computer; see, e.g., [1] for more details on binomial models.

\subsection{Put-Call parity}

In the previous subsection I only considered the price of a (European) call option, and the attentive reader might have wondered how can one determine the price of the corresponding put option. It turns out that there is a simple relationship between European put and call options, so that from the price of one of them we can obtain the price of the other. To see this, form the following portfolio: i) buy one stock $S$ and one put option $P$ on this stock with strike price $K$ and maturity $T$, and ii) short one call option $C$ with the same strike and maturity as the put option. The value of such portfolio would thus be

$$
V=S+P-C .
$$

Now from (5) and (6), one immediately sees that at expiration we have $P-C=K-S$, so that the value of the above portfolio at time $T$ becomes simply

$$
V_{T}=K \text {. }
$$

Since this portfolio has a known (i.e., riskless) value at time $t=T$, it then follows from the no-arbitrage condition that its value at any time $0 \leq t \leq T$ must be given by

$$
V=K e^{-r(T-t)},
$$

where $r$ is the risk-free interest rate. Inserting (13) into (11) immediately yields the so-called put-call parity relation:

$$
P=C-S+K e^{-r(T-t)} .
$$

\section{Brownian motion and stochastic calculus}

\subsection{One-dimensional random walk}

Every physics graduate student is familiar, in one way or another, with the concept of a Brownian motion. The customary introduction [15] to this subject is through the notion of a random walk, in which the anecdotal drunk walks along a line taking at every time interval $\Delta t$ one step of size $l$, either to the right or to the left with equal probability. The position, $X(t)$, of the walker after a time $t=N \Delta t$, where $N$ is the number of steps taken, represents a stochastic process. (See Appendix A for a formal definition of random variables and stochastic processes.) As is well known, the probability $P(X(t)=x)$ for the walker to be found at a given position $x=n l$, where $n$ is an integer, at given time $t$, is described by a binomial distribution [15].

Simply stated, the Brownian motion is the stochastic process that results by taking the random walk to the continuous limit: $\Delta t \rightarrow 0, l \rightarrow 0, N \rightarrow \infty, n \rightarrow \infty$ such that $t=N \Delta t$ and $x=n l$ remain finite. (A more formal definition is given below.) Here, however, some caution with the limits must be taken to ensure that a finite probability density $p(x, t)$ is obtained: one must take $\Delta t \rightarrow 0$ and $l \rightarrow 0$, such that $l^{2}=\sigma \Delta t$, where $\sigma$ is a constant. In this case one obtains that $p(x, t)$ is given by a Gaussian distribution [15]:

$$
p(x, t)=\frac{1}{\sqrt{2 \pi \sigma^{2} t}} \exp \left\{-\frac{x^{2}}{2 \sigma^{2} t}\right\} .
$$

At this point let us establish some notation. Let $X$ be a random variable with probability density function (pdf) given by $p(x)$. [Following standard practice, we shall denote a random variable by capital letters, while the values it takes will be written in small letters]. The operator for expectation value will be denoted either as $E[\cdot]$ or $\langle\cdot\rangle$, that is,

$$
E[f(X)] \equiv\langle f(X)\rangle=\int_{-\infty}^{\infty} f(x) p(x) d x
$$

where $f(x)$ is an arbitrary function. Although the angularbracket notation for expectation value is preferred by physicists, we shall often use the $E$ notation which is more convenient for our purposes. 
A Gaussian or normal distribution with mean $m$ and standard deviation $\sigma$ will be denoted by $\mathcal{N}(m, \sigma)$, whose pdf is

$$
p_{\mathcal{N}}(x, t)=\frac{1}{\sqrt{2 \pi \sigma^{2}}} \exp \left\{-\frac{(x-m)^{2}}{2 \sigma^{2}}\right\} .
$$

Let us also recall that the (nonzero) moments of the Gaussian distribution are as follows

$$
\begin{aligned}
E[X] & =m, \quad E\left[X^{2}\right]=\sigma^{2}, \\
E\left[X^{2 n}\right] & =1 \cdot 3 \cdot 5 \cdot \ldots \cdot(2 n-1) \sigma^{2 n} .
\end{aligned}
$$

\subsection{Brownian motion and white noise}

We have seen above that a 1D Brownian motion can be thought of as the limit of a random walk after infinitely many infinitesimal steps. This formulation was first given in 1900 by Bachelier [14] who also showed the connection between Brownian motion and the diffusion equation (five years before Einstein's famous work on the subject [16]). It is thus telling that the first theory of Brownian motion was developed to model financial asset prices! A rigorous mathematical theory for the Brownian motion was constructed by Wiener [17] in 1923, after which the Brownian motion became also known as the Wiener process.

Definition 2 The standard Brownian motion or Wiener process $\{W(t), t \geq 0\}$ is a stochastic process with the following properties:

1. $W(0)=0$.

2. The increments $W(t)-W(s)$ are stationary and independent.

3. For $t>s, W(t)-W(s)$ has a normal distribution $\mathcal{N}(0, \sqrt{t-s})$

\section{The trajectories are continuous (i.e., "no jumps").}

The stationarity condition implies that the pdf of $W(t)-$ $W(s)$, for $t>s$, depends only on the time difference $t-s$.
(For a more precise definition of stationary processes see Appendix A.) Now, it is not hard to convince oneself that conditions 2 and 3 imply that $W(t)$ is distributed according to $\mathcal{N}(0, \sqrt{t})$ for $t>0$. In particular, we have $E[W(t)]=0$ for all $t \geq 0$. Furthermore, one can easily show that the covariance of the Brownian motion is given by

$$
E[W(t) W(s)]=s, \quad \text { for } \quad t>s .
$$

It is also clear from the definition above that the Brownian motion is a Gaussian process (see Appendix A for the formal definition of Gaussian processes). Then, since a Gaussian process is fully characterized by its mean and covariance, we can give the following alternative definition of the Brownian motion.

Definition 3 The standard Brownian motion or Wiener process $\{W(t), t \geq 0\}$ is a Gaussian process with $E[W(t)]=$ 0 and $E[W(t) W(s)]=\min (s, t)$.

The Brownian motion has the important property of having bounded quadratic variation. To see what this means, consider a partition $\left\{t_{i}\right\}_{i=0}^{n}$ of the interval $[0, t]$, where $0=t_{0}<t_{1}<\ldots<t_{n}=t$. For simplicity, let us take equally spaced time intervals: $t_{i}-t_{i-1}=\Delta t=\frac{t}{n}$. The quadratic variation of $W(t)$ on $[0, t]$ is defined as

$$
Q_{n}=\sum_{i=0}^{n} \Delta W_{i}^{2}
$$

where $\Delta W_{i}=W\left(t_{i}\right)-W\left(t_{i-1}\right)$. Since $\Delta W_{i}$ is distributed according to $\mathcal{N}(0, \sqrt{\Delta t})$ we have that $E\left[\Delta W^{2}\right]=\Delta t$, which implies that

$$
E\left[Q_{n}\right]=t
$$

Furthermore, using the fact that the increments $\Delta W_{i}$ are independent and recalling that the variance of the sum of independent variables is the sum of the variances, we get for the variance of $Q_{n}$ :

$$
\begin{aligned}
\operatorname{var}\left[\mathrm{Q}_{\mathrm{n}}\right] & =\sum_{i=0}^{n} \operatorname{var}\left[\Delta W_{i}^{2}\right]=\sum_{i=0}^{n}\left\{E\left[\Delta W_{i}^{4}\right]-\left(E\left[\Delta W_{i}^{2}\right]\right)^{2}\right\} \\
& =\sum_{i=0}^{n}\left[3(\Delta t)^{2}-(\Delta t)^{2}\right]=\frac{2 t^{2}}{n},
\end{aligned}
$$

where in the third equality we used (19) and the fact that $\Delta W_{i}$ has distribution $\mathcal{N}(0, \sqrt{\Delta t})$. We thus see that

$$
\operatorname{var}\left[\mathrm{Q}_{\mathrm{n}}\right] \rightarrow 0, \quad \text { as } \quad \mathrm{n} \rightarrow \infty
$$

On the other hand, we have that

$$
\operatorname{var}\left[\mathrm{Q}_{\mathrm{n}}\right]=\mathrm{E}\left[\left(\mathrm{Q}_{\mathrm{n}}-\mathrm{E}\left[\mathrm{Q}_{\mathrm{n}}\right]\right)^{2}\right]=\mathrm{E}\left[\left(\mathrm{Q}_{\mathrm{n}}-\mathrm{t}\right)^{2}\right]
$$

where in the last equality we have used (21). Comparing 
(22) and (23) then yields

$$
\lim _{n \rightarrow \infty} E\left[\left(Q_{n}-t\right)^{2}\right]=0 .
$$

We have thus proven that $Q_{n}$ converges to $t$ in the mean square sense. This fact suggests that $\Delta W^{2}$ can be thought of as being of the order of $\Delta t$, meaning that as $\Delta t \rightarrow 0$ the quantity $\Delta W^{2}$ "resembles more and more" the deterministic quantity $\Delta t$. In terms of differentials, we write

$$
[d W]^{2}=d t .
$$

Alternatively, we could say that $d W$ is of order $\sqrt{d t}$ :

$$
d W=O(\sqrt{d t}) .
$$

(I remark parenthetically that the boundedness of the quadratic variation of the Brownian motion should be contrasted with the fact that its total variation, $A_{n}=$ $\sum_{i=0}^{n}\left|\Delta W_{i}\right|$, is unbounded, that is, $A_{n} \rightarrow \infty$ as $n \rightarrow \infty$, with probability 1 ; see [7].)

Another important property of the Brownian motion $W(t)$ is the fact that it is self-similar (or more exactly selfaffine) in the following sense:

$$
W(a t) \stackrel{d}{=} a^{1 / 2} W(t),
$$

for all $a>0$. Here $\stackrel{d}{=}$ means equality in the sense of probability distribution, that is, the two processes $W(a t)$ and $a^{1 / 2} W(t)$ have exactly the same finite-dimensional distributions $p\left(x_{1}, t_{1} ; \ldots, x_{n}, t_{n}\right)$ for any choice of $t_{i}, i=1, \ldots, n$, and $n \geq 1$. Self-similarity means that any finite portion of a Brownian motion path when properly rescaled is (statistically) indistinguishable from the whole path. For example, if we 'zoom in' in any given region (no matter how small) of a Brownian motion path, by rescaling the time axis by a factor of $a$ and the vertical axis by a factor of $\sqrt{a}$, we obtain a curve similar (statistically speaking) to the original path. An example of this is shown in Fig. 7. In the language of fractals, we say that a trajectory of a Brownian motion is a fractal curve with fractal dimension $D=2$.

The self-similarity property implies that sample paths of a Brownian motion are nowhere differentiable (technically, with probability 1). A formal proof of this fact, although not difficult, is beyond the scope of the present notes, so that here we shall content ourselves with the following heuristic argument. Suppose we try to compute the derivative of $W(t)$ in the usual sense, that is,

$$
\frac{d W}{d t}=\lim _{\Delta t \rightarrow 0} \frac{\Delta W}{\Delta t}=\lim _{\Delta t \rightarrow 0} \frac{W(t+\Delta t)-W(t)}{\Delta t} .
$$

But since $\Delta W$ is of order $\sqrt{\Delta t}$, it then follows that

$$
\frac{\Delta W}{\Delta t}=O\left(\frac{1}{\sqrt{\Delta t}}\right)
$$

so that $d W / d t=\infty$ as $\Delta t \rightarrow 0$.
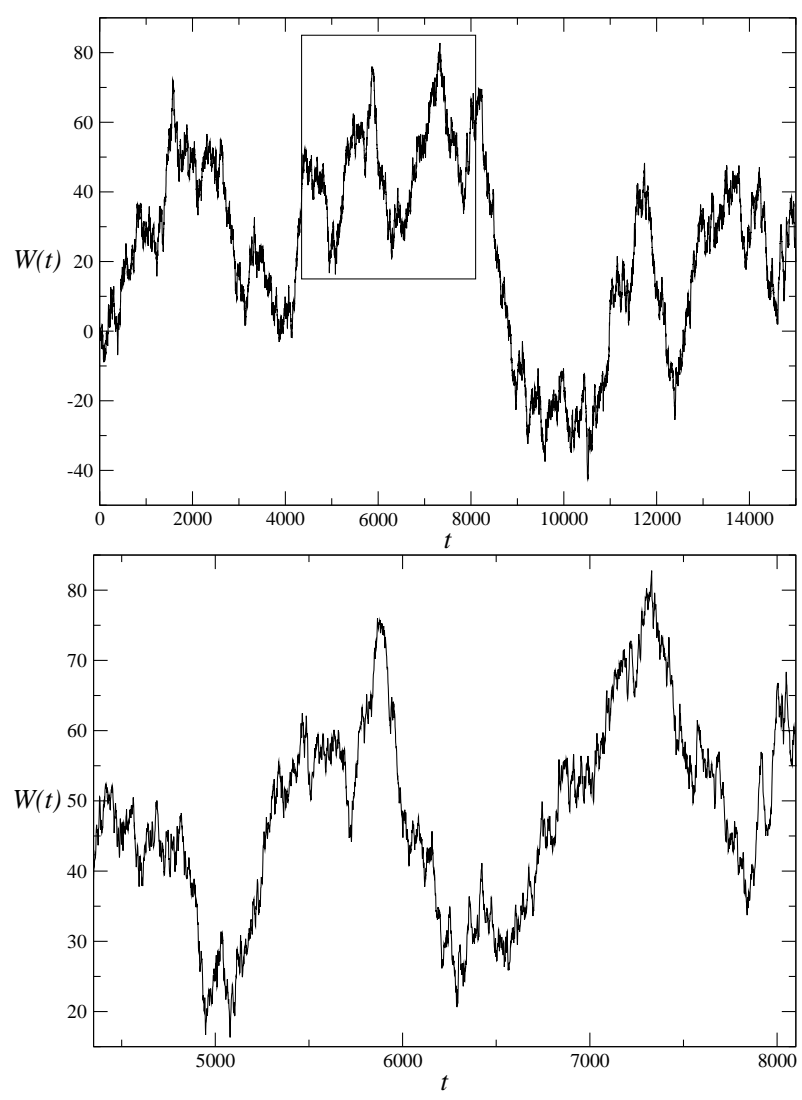

Figure 7. Self-similarity of a Brownian motion path. In (a) we plot a path of a Brownian motion with 15000 time steps. The curve in (b) is a blow-up of the region delimited by a rectangle in (a), where we have rescaled the $x$ axis by a factor 4 and the $y$ axis by a factor 2. Note that the graphs in (a) and (b) "look the same," statistically speaking. This process can be repeated indefinitely.

Although the derivative of $W(t)$ does not exist as a regular stochastic process, it is possible to give a mathematical meaning to $d W / d t$ as a generalized process (in the sense of generalized functions or distributions). In this case, the derivative of the $W(t)$ is called the white noise process $\xi(t)$ :

$$
\xi(t) \equiv \frac{d W}{d t} .
$$

I shall, of course, not attempt to give a rigorous definition of the white noise, and so the following intuitive argument will suffice. Since according to (27) the derivative $\frac{d W}{d t}$ diverges as $\frac{1}{\sqrt{d t}}$, a simple power-counting argument suggests that integrals of the form

$$
I(t)=\int_{0}^{t} g\left(t^{\prime}\right) \xi\left(t^{\prime}\right) d t^{\prime},
$$

should converge (in some sense); see below.

In physics, the white noise $\xi(t)$ is simply 'defined' as a 'rapidly fluctuating function' [15] (in fact, a generalized stochastic process) that satisfies the following conditions

$$
\begin{aligned}
\langle\xi(t)\rangle & =0, \\
\left\langle\xi(t) \xi\left(t^{\prime}\right)\right\rangle & =\delta\left(t-t^{\prime}\right) .
\end{aligned}
$$


These two relations give the 'operational rules' from which quantities such as the mean and the variance of the integral $I(t)$ in (29) can be calculated. It is convenient, however, to have an alternative definition of stochastic integrals in terms of regular stochastic process. Such a construction was first given by the Japanese mathematician Itô [18].

\subsection{Itô stochastic integrals}

Using (28), let us first rewrite integral (29) as an "integral over the Wiener process" $W(t)$ :

$$
I(t)=\int_{0}^{t} g\left(t^{\prime}\right) d W\left(t^{\prime}\right) .
$$

The idea then is to define this integral as a kind of RiemannStieltjes integral. We thus take a partition $\left\{t_{i}\right\}_{i=0}^{n}$ of the interval $[0, t]$ and consider the partial sums

$$
I_{n}=\sum_{i=1}^{n} g\left(t_{i-1}\right) \Delta W\left(t_{i}\right) \equiv \sum_{i=1}^{n} g\left(t_{i-1}\right)\left[W\left(t_{i}\right)-W\left(t_{i-1}\right)\right] .
$$

The function $g(t)$ above must satisfy certain appropriate conditions [7], the most important one being that $g(t)$ be a non-anticipating function. This means, in particular, that the value $g\left(t_{i-1}\right)$ in (33) is independent of the 'next increment' $\Delta W\left(t_{i}\right)$ of the Brownian motion. [For this reason, choosing to evaluate $g(t)$ at the beginning of the interval $\Delta t_{i}=t_{i}-t_{i-1}$ is a crucial point in the definition of the Itô stochastic integral. Another possible choice is to evaluate $g(t)$ at the mid point $t^{*}=\left(t_{i-1}+t_{i}\right) / 2$, which leads to the Stratonovich integral [8]. In these notes I shall only consider Itô integrals.]

Under the appropriate conditions on $g(t)$, it is then possible to show that the partial sums $I_{n}$ converge in the mean square sense. That is, there exists a process $I(t)$ such that

$$
E\left[\left(I_{n}-I(t)\right)^{2}\right] \rightarrow 0 \quad \text { as } \quad n \rightarrow \infty
$$

Using the fact that $g(t)$ is non-anticipating and that $E[\Delta W(t)]=0$, it follows immediately from the definition (33) that $I(t)$ has zero mean:

$$
E[I(t)]=E\left[\int_{0}^{t} g\left(t^{\prime}\right) d W\left(t^{\prime}\right)\right]=0,
$$

It is also possible to show that stochastic integrals obey the so-called isometry property:

$$
\begin{aligned}
E\left[\{I(t)\}^{2}\right] & =E\left[\left(\int_{0}^{t} g\left(t^{\prime}\right) d W\left(t^{\prime}\right)\right)^{2}\right] \\
& =\int_{0}^{t} E\left[g^{2}\left(t^{\prime}\right)\right] d t^{\prime} .
\end{aligned}
$$

We now see that the true meaning of conditions (30) and (31) is given by properties (35) and (36), for the particular case when $g(t)$ is a deterministic function.

The Itô integral does not conform to the usual integration rules from deterministic calculus. An example is the formula below

$$
\int_{0}^{t} W d W=\frac{1}{2} W(t)^{2}-\frac{1}{2} t
$$

which is left as an exercise for the reader [19]. Itô integrals offer however a convenient way to define (and deal with) stochastic differential equations, as we will see next.

\subsection{Stochastic differential equations}

Physicists are quite familiar with differential equations involving stochastic terms, such as the Langevin equation

$$
\frac{d v}{d t}=-\gamma v+\sigma \xi(t)
$$

which describes the motion of a Brownian particle in a viscous liquid [15]. Here $\gamma$ is the viscosity of the fluid and $\sigma$ is the 'amplitude' of the fluctuating force acting on the Brownian particle. (These parameters are usually considered to be constant but in general they could be non-anticipating functions of time.) Equation (37) does not however make much mathematical sense, since it evolves a quantity, namely, the derivative $\xi(t)$ of the Brownian motion, that does not even exist (except as a generalized process). Nevertheless, it is possible to put this equation on a firm mathematical basis by expressing it as a stochastic integral equation. First we rewrite (37) as

$$
d v=-\gamma v d t+\sigma d W
$$

which upon integration yields

$$
v(t)=v(0)-\int_{0}^{t} \gamma v\left(t^{\prime}\right) d t^{\prime}+\int_{0}^{t} \sigma d W\left(t^{\prime}\right) .
$$

This integral equation now makes perfectly good sense-in fact, its solution can be found explicitly [19].

Let us now consider more general stochastic differential equations (SDE) of the form

$$
d X=a(X, t) d t+b(X, t) d W
$$


where $a(x, t)$ and $B(x, t)$ are known functions. Note that this 'differential equation' is actually a short-hand notation for the following stochastic integral equation

$$
X(t)=X(0)+\int_{0}^{t} a\left(X, t^{\prime}\right) d t^{\prime}+\int_{0}^{t} b\left(X, t^{\prime}\right) d W\left(t^{\prime}\right)
$$

Under certain condition on the functions $a(x, t)$ and $b(x, t)$, it is possible to show (see, e.g., [8]) that the SDE (40) has a unique solution $X(t)$.

Let us discuss another simple SDE, namely, the Brownian motion with drift:

$$
d X=\mu d t+\sigma d W
$$

where the constant $\mu$ represents the mean drift velocity. Integrating (42) immediately yields the process

$$
X(t)=\mu t+W(t),
$$

whose pdf is

$$
p(x, t)=\frac{1}{2 \pi \sigma^{2} t} \exp \left\{\frac{(x-\mu t)^{2}}{2 \sigma^{2} t}\right\} .
$$

Another important example of a (linear) SDE that can be solved explicitly is the geometric Brownian motion that will be discussed shortly. But before doing that, let us discuss a rather useful result known as Itô lemma or Itô formula.

\subsection{Itô formula}

Consider the generic process $X(t)$ described by the SDE (40), and suppose that we have a new stochastic process $Z$ defined by

$$
Z(t)=F(X(t), t),
$$

for some given function $F(x, t)$. We now wish to find the local dynamics followed by the $Z(t)$, that is, the SDE whose solutions corresponds to the process $Z(t)$ above. The answer is given by the Itô formula that we now proceed to derive.

First, consider the Taylor expansion of the function $F(X, t)$ :

$$
\begin{aligned}
d F= & \frac{\partial F}{\partial t} d t+\frac{\partial F}{\partial x} d X+\frac{1}{2} \frac{\partial^{2} F}{\partial x^{2}}(d X)^{2}+ \\
& +\frac{1}{2} \frac{\partial^{2} F}{\partial t^{2}}(d t)^{2}+\frac{1}{2} \frac{\partial^{2} F}{\partial t \partial x} d t d X+\ldots
\end{aligned}
$$

Note, however, that

$$
\begin{aligned}
(d X)^{2} & =b^{2} d W^{2}+2 a b d t d W+a^{2}(d t)^{2} \\
& =b^{2} d t+O\left(d t^{3 / 2}\right)
\end{aligned}
$$

where we used the fact that $d W^{2}=d t$ and $d t d W=$ $O\left(d t^{3 / 2}\right)$. (Here we have momentarily omitted the arguments of the functions $a$ and $b$ for ease of notation.) Inserting (47) into (46) and retaining only terms up to order $d t$, we obtain

$$
d F=\left[\frac{\partial F}{\partial t}+\frac{1}{2} b^{2} \frac{\partial^{2} F}{\partial x^{2}}\right] d t+b \frac{\partial F}{\partial x} d X,
$$

which is known as Itô formula. Upon using (40) in the equation above, we obtain Itô formula in a more explicit fashion

$$
\begin{aligned}
d F= & {\left[\frac{\partial F}{\partial t}+a(X, t) \frac{\partial F}{\partial x}+\frac{1}{2} b^{2}(X, t) \frac{\partial^{2} F}{\partial x^{2}}\right] d t } \\
+\quad & b(X, t) \frac{\partial F}{\partial x} d W
\end{aligned}
$$

What is noteworthy about this formula is the fact that the fluctuating part of the primary process $X(t)$ contributes to the drift of the derived process $Z(t)=F(t, X)$ through the term $\frac{1}{2} b^{2}(t, X) \frac{\partial^{2} F}{\partial x^{2}}$. We shall next use Itô formula to solve explicitly a certain class of linear SDE's.

\subsection{Geometric Brownian motion}

A stochastic process of great importance in Finance is the so-called geometric Brownian notion, which is defined as the solution to the following SDE

$$
d S=\mu S d t+\sigma S d W
$$

where $\mu$ and $\sigma$ are constants, subjected to a generic initial condition $S\left(t_{0}\right)=S_{0}$. Let us now perform the following change of variables $Z=\ln S$. Applying Itô formula (49) with $a=\mu S, b=\sigma S$ and $F(S)=\ln S$, it then follows that

$$
d Z=\left(\mu-\frac{1}{2} \sigma^{2}\right) d t+\sigma d W
$$

which upon integration yields

$$
Z(t)=Z_{0}+\left(\mu-\frac{1}{2} \sigma^{2}\right)\left(t-t_{0}\right)+\sigma\left[W(t)-W\left(t_{0}\right)\right]
$$

where $Z_{0}=\ln S_{0}$. Reverting to the variable $S$ we obtain the explicit solution of the SDE (50):

$S(t)=S_{0} \exp \left\{\left(\mu-\frac{1}{2} \sigma^{2}\right)\left(t-t_{0}\right)+\sigma\left[W(t)-W\left(t_{0}\right)\right]\right\}$.

From (52) we immediately see that $Z(t)-Z_{0}$ is distributed according to $\mathcal{N}\left(\left(\mu-\frac{1}{2} \sigma^{2}\right) \tau, \sigma \sqrt{\tau}\right)$, where $\tau=$ $t-t_{0}$. It then follows that the geometric Brownian motion with initial value $S\left(t_{0}\right)=S_{0}$ has the following log-normal distribution: 


$$
p\left(S, t ; S_{0}, t_{0}\right)=\frac{1}{\sqrt{2 \sigma^{2} \tau} S} \exp \left\{-\frac{\left[\ln \left(\frac{S}{S_{0}}\right)-\left(\mu-\frac{1}{2} \sigma^{2}\right) \tau\right]^{2}}{2 \sigma^{2} \tau}\right\}
$$

The geometric Brownian motion is the basic model for stock price dynamics in the Black-Scholes framework, to which we now turn.

\section{The Standard Model of Finance}

\subsection{Portfolio dynamics and arbitrage}

Consider a financial market with only two assets: a riskfree bank account $B$ and a stock $S$. In vector notation, we write $\vec{S}(t)=(B(t), S(t))$ for the asset price vector at time $t$. A portfolio in this market consists of having an amount $x_{0}$ in the bank and owing $x_{1}$ stocks. The vector $\vec{x}(t)=\left(x_{0}(t), x_{1}(t)\right)$ thus describes the time evolution of your portfolio in the $(B, S)$ space. Note that $x_{i}<0$ means a short position on the $i$ th asset, i.e., you 'owe the market' $\left|x_{i}\right|$ units of the $i$ th asset. Let us denote by $V_{\vec{x}}(t)$ the money value of the portfolio $\vec{x}(t)$ :

$$
V_{\vec{x}}=\vec{x} \cdot \vec{S}=x_{0} B+x_{1} S,
$$

where the time dependence has been omitted for clarity. We shall also often suppress the subscript from $V_{\vec{x}}(t)$ when there is no risk of confusion about to which portfolio we are referring.

A portfolio is called self-financing if no money is taken from it for 'consumption' and no additional money is invested in it, so that any change in the portfolio value comes solely from changes in the asset prices. More precisely, a portfolio $\vec{x}$ is self-financing if its dynamics is given by

$$
d V_{\vec{x}}(t)=\vec{x}(t) \cdot d \vec{S}(t), \quad t \geq 0 .
$$

The reason for this definition is that in the discrete-time case, i.e., $t=t_{n}, n=0,1,2, \ldots$, the increase in wealth, $\Delta V\left(t_{n}\right)=V\left(t_{n+1}\right)-V\left(t_{n}\right)$, of a self-financing portfolio over the time interval $t_{n+1}-t_{n}$ is given by

$$
\Delta V\left(t_{n}\right)=\vec{x}\left(t_{n}\right) \cdot \Delta \vec{S}\left(t_{n}\right),
$$

where $\Delta \vec{S}\left(t_{n}\right) \equiv \vec{S}\left(t_{n+1}\right)-\vec{S}\left(t_{n}\right)$. This means that over the time interval $t_{n+1}-t_{n}$ the value of the portfolio varies only owing to the changes in the asset prices themselves, and then at time $t_{n+1}$ re-allocate the assets within the portfolio for the next time period. Equation (56) generalizes this idea for the continuous-time limit. If furthermore we decide on the make up of the portfolio by looking only at the current prices and not on past times, i.e., if

$$
\vec{x}(t)=\vec{x}(t, \vec{S}(t)),
$$

then the portfolio is said to be Markovian. Here we shall deal exclusively with Markovian portfolios.

As we have seen already in Sec. 2.4, an arbitrage represents the possibility of making a riskless profit with no initial commitment of money. A more formal definition of arbitrage is as follows.

Definition 4 An arbitrage is a portfolio whose value $V(t)$ obeys the following conditions

(i) $V(0)=0$

(ii) $V(t) \geq 0$ with probability 1 for all $t>0$

(iii) $V(T)>0$ with positive probability for some $T>0$.

The meaning of the first condition is self-evident. The second condition says that there is no chance of losing money, while the third one states that there is a possibility that the portfolio will acquire a positive value at some time $T$. Thus, if you hold this portfolio until this arbitrage time there is a real chance that you will make a riskless profit out of nothing. [If $P(V(T)>0)=1$ we have a strong arbitrage opportunity, in which case we are sure to make a profit.] As we have already discussed in Sec. 2.4, arbitrage opportunities are very rare and can last only for a very short time (typically, of the order of seconds or a few minutes at most). In fact, in the famous Black-Scholes model that we will now discuss it is assumed that there is no arbitrage at all.

\subsection{The Black-Scholes model for option pric- ing}

The two main assumptions of the Black-Scholes model are:

(i) There are two assets in the market, a bank account $B$ and a stock $S$, whose price dynamics are governed by the following differential equations

$$
\begin{aligned}
d B & =r B d t \\
d S & =\mu S d t+\sigma S d W
\end{aligned}
$$

where $r$ is the risk-free interest rate, $\mu>0$ is the stock mean rate of return, $\sigma>0$ is the volatility, and $W(t)$ is the standard Brownian motion or Wiener process.

(ii) The market is free of arbitrage. 
Besides these two crucial hypothesis, there are additional simplifying (technical) assumptions, such as: (iii) there is a liquid market for the underlying asset $S$ as well as for the derivative one wishes to price, (iv) there are no transaction costs (i.e., no bid-ask spread), and (v) unlimited short selling is allowed for an unlimited period of time. It is implied by (58) that there is no interest-rate spread either, that is, money is borrowed and lent at the same rate $r$. Equation (59) also implies that the stock pays no dividend. [This last assumption can be relaxed to allow for dividend payments at a known (i.e., deterministic) rate; see, e.g., [4] for details.]

We shall next describe how derivatives can be 'rationally' priced in the Black-Scholes model. We consider first a European call option for which a closed formula can be found. (More general European contingent claims will be briefly considered at the end of the Section.) Let us then denote by $C(S, t ; K, T)$ the present value of a European call option with strike price $K$ and expiration date $T$ on the underlying stock $S$. For ease of notation we shall drop the parameters $K$ and $T$ and simply write $C(S, t)$. For later use, we note here that according to Itô formula (49), with $a=\mu S$ and $b=\sigma S$, the option price $C$ obeys the following dynamics

$$
d C=\left[\frac{\partial C}{\partial t}+\mu S \frac{\partial C}{\partial S}+\frac{1}{2} \sigma^{2} S^{2} \frac{\partial^{2} C}{\partial S^{2}}\right] d t+\sigma S \frac{\partial C}{\partial S} d W .
$$

In what follows, we will arrive at a partial differential equation, the so-called Black-Scholes equation (BSE), for the option price $C(S, t)$. For pedagogical reasons, we will present two alternative derivations of the BSE using two distinct but related arguments: i) the $\Delta$-hedging portfolio and ii) the replicating portfolio.

\subsubsection{The delta-hedging portfolio}

As in the binomial model of Sec. 2.5, we consider the selffinancing $\Delta$-hedging portfolio, consisting of a long position on the option and a short position on $\Delta$ stocks. The value $\Pi(t)$ of this portfolio is

$$
\Pi(t)=C(S, t)-\Delta S .
$$

Since the portfolio is self-financing, it follows from (56) that $\Pi$ obeys the following dynamics

$$
d \Pi=d C-\Delta d S,
$$

which in view of (59) and (60) becomes

$$
\begin{aligned}
d \Pi= & {\left[\frac{\partial C}{\partial t}+\mu S \frac{\partial C}{\partial S}+\frac{1}{2} \sigma^{2} S^{2} \frac{\partial^{2} C}{\partial S^{2}}-\mu \Delta S\right] d t } \\
& +\sigma S\left(\frac{\partial C}{\partial S}-\Delta\right) d W .
\end{aligned}
$$

We can now eliminate the risk [i.e., the stochastic term containing $d W]$ from this portfolio by choosing

$$
\Delta=\frac{\partial C}{\partial S}
$$

Inserting this back into (62), we then find

$$
d \Pi=\left[\frac{\partial C}{\partial t}+\frac{1}{2} \sigma^{2} S^{2} \frac{\partial^{2} C}{\partial S^{2}}\right] d t .
$$

Since we now have a risk-free (i.e., purely deterministic) portfolio, it must yield the same rate of return as the bank account, which means that

$$
d \Pi=r \Pi d t
$$

Comparing (64) with (65) and using (61) and (63), we then obtain the Black-Scholes equation:

$$
\frac{\partial C}{\partial t}+\frac{1}{2} \sigma^{2} S^{2} \frac{\partial^{2} C}{\partial S^{2}}+r S \frac{\partial C}{\partial S}-r C=0,
$$

which must be solved subjected to the following boundary condition

$$
C(S, T)=\max (S-K, 0) .
$$

The solution to the above boundary-value problem can be found explicitly (see below), but before going into that it is instructive to consider an alternative derivation of the BSE. [Note that the above derivation of the BSE remains valid also in the case that $r, \mu$, and, $\sigma$ are deterministic functions of time, although a solution in closed form is no longer possible.]

\subsubsection{The replicating portfolio}

Here we will show that it is possible to form a portfolio on the $(B, S)$ market that replicates the option $C(S, t)$, and in the process of doing so we will arrive again at the BSE. Suppose then that there is indeed a self-financing portfolio $\vec{x}(t)=(x(t), y(t))$, whose value $Z(t)$ equals the option price $C(S, t)$ for all time $t \leq T$ :

$$
Z \equiv x B+y S=C
$$

where we have omitted the time-dependence for brevity. Since the portfolio is self-financing it follows that

$$
d Z=x d B+y d S=(r x B+\mu y S) d t+\sigma y S d W .
$$

But by assumption we have $Z=C$ and so $d Z=d C$. Comparing (69) with (60) and equating the coefficients separately in both $d W$ and $d t$, we obtain

$$
\begin{aligned}
& y=\frac{\partial C}{\partial S}, \\
& \frac{\partial C}{\partial t}-r x B+\frac{1}{2} \sigma^{2} S^{2} \frac{\partial^{2} C}{\partial S^{2}}=0 .
\end{aligned}
$$

Now from (68) and (70) we get that

$$
x=\frac{1}{B}\left[C-S \frac{\partial C}{\partial S}\right]
$$


which inserted into (71) yields again the BSE (66), as the reader can easily verify.

We have thus proven, by direct construction, that the option $C$ can be replicated in the $(B, S)$-market by the portfolio $(x, y)$, where $x$ and $y$ are given in (72) and (70), respectively, with option price $C$ being the solution of the BSE (with the corresponding boundary condition). [To complete the proof, we must also show that the initial price $C_{0}=C(S, 0)$ is the 'correct' one, in the sense that if the option price were $C_{0}^{\prime} \neq C_{0}$, then there would be an arbitrage opportunity. In fact, if $C_{0}^{\prime}>C_{0}$ an arbitrageur should short the option and invest in the replicating portfolio, whereas if $C_{0}^{\prime}<C_{0}$ he should do the opposite.]

\subsection{The Black-Scholes formula}

Here we will solve equation (66) subjected to the boundary condition (67). Following the original work of Black and Scholes [12], the idea is to perform a change of variables so as to turn the BSE into the heat equation, which we know how to solve. Here we will not use the original transformation employed by these authors but a related one [6], as shown below:

$$
\begin{gathered}
\tau=\frac{T-t}{2 / \sigma^{2}}, \quad x=\ln \left(\frac{S}{K}\right), \\
u(x, \tau)=e^{\alpha x+\beta^{2} \tau} \frac{C(S, t)}{K},
\end{gathered}
$$

where

$$
\alpha=\frac{1}{2}\left(\frac{2 r}{\sigma^{2}}-1\right), \beta=\frac{1}{2}\left(\frac{2 r}{\sigma^{2}}+1\right) .
$$

After a somewhat tedious but straightforward algebra [6], one obtains that in the new variables equation (66) reads

$$
\frac{\partial u}{\partial \tau}=\frac{\partial^{2} u}{\partial x^{2}}
$$

while the terminal condition (67) becomes an initial condition

$$
u(x, 0)=u_{0}(x)=\max \left(e^{\beta x}-e^{\alpha x}, 0\right) .
$$

We now recall that the Green's function for the heat equation is

$$
G\left(x, x^{\prime}\right)=\frac{1}{\sqrt{4 \pi \tau}} e^{-\left(x-x^{\prime}\right)^{2} / 4 \tau},
$$

so that its generic solution for an arbitrary initial condition $u_{0}(x)$ is given by

$$
\begin{aligned}
u(x, \tau) & =\int_{-\infty}^{\infty} u_{0}\left(x^{\prime}\right) G\left(x, x^{\prime}\right) d x^{\prime} \\
& =\frac{1}{\sqrt{4 \pi \tau}} \int_{-\infty}^{\infty} u_{0}\left(x^{\prime}\right) e^{-\left(x-x^{\prime}\right)^{2} / 4 \tau} d x^{\prime}
\end{aligned}
$$

Inserting (77) into the integral above we obtain

$$
u(\tau, x)=\frac{1}{\sqrt{4 \pi \tau}} \int_{0}^{\infty}\left(e^{\beta x^{\prime}}-e^{\alpha x^{\prime}}\right) e^{-\left(x-x^{\prime}\right)^{2} / 4 \tau} d x^{\prime}
$$

$$
=I(\beta)-I(\alpha)
$$

where

$$
I(a) \equiv \frac{1}{\sqrt{4 \pi \tau}} \int_{0}^{\infty} e^{a x^{\prime}} e^{-\left(x-x^{\prime}\right)^{2} / 4 \tau} d x^{\prime} .
$$

After completing the squares and performing some simplification, we find that

$$
I(a)=e^{a x+a^{2} \tau} N\left(d_{a}\right)
$$

where

$$
d_{a}=\frac{x+2 a \tau}{\sqrt{2 \tau}}
$$

and $N(x)$ denotes the cumulative distribution function for a normal variable $\mathcal{N}(0,1)$ :

$$
N(x)=\frac{1}{\sqrt{2 \pi}} \int_{-\infty}^{x} e^{-s^{2} / 2} d s .
$$

Inserting (81) into (79) and reverting back to the original dimensional variables, we obtain the famous Black-Scholes formula for the price of a European call option:

$$
C(S, t)=S N\left(d_{1}\right)-K e^{-r(T-t)} N\left(d_{2}\right),
$$

where

$$
\begin{aligned}
& d_{1}=\frac{\ln \left(\frac{S}{K}\right)+\left(r+\frac{1}{2} \sigma^{2}\right)(T-t)}{\sigma \sqrt{T-t}}, \\
& d_{2}=\frac{\ln \left(\frac{S}{K}\right)+\left(r-\frac{1}{2} \sigma^{2}\right)(T-t)}{\sigma \sqrt{T-t}} .
\end{aligned}
$$

This formula is so often used in practice that it is already pre-defined in many software packages (e.g., Excel, Matlab, Maple, etc) as well as in most modern hand calculators with financial functions. It should noted, however, that many people (academics and practitioners alike) believe that the Black-Scholes model is too idealized to describe real market situations; see Secs. V and VII for a brief discussion of possible extensions of the BS model.

\subsection{Completeness in the Black-Scholes model}

We have seen above that it is possible to replicate a European call option $C(S, t)$ using an appropriate self-financing portfolio in the $(B, S)$ market. Looking back at the argument given in Sec. 4.2.2, we see that we never actually made use of the fact that the derivative in question was a call option-the nature of the derivative appeared only through the boundary condition (67). Thus, the derivation of the BSE presented there must hold for any contingent claim!

To state this fact more precisely, let $F(S, t)$ represent the price of an arbitrary European contingent claim with payoff $F(S, T)=\Phi(S)$, where $\Phi$ is a known function. Retracing the steps outlined in Sec. 4.2.2, we immediately conclude 
that the price $F(S, t)$ will be the solution to the following boundary-value problem

$$
\begin{array}{r}
\frac{\partial F}{\partial t}+\frac{1}{2} \sigma^{2} S^{2} \frac{\partial^{2} F}{\partial S^{2}}+r S \frac{\partial F}{\partial S}-r F=0 \\
F(S, T)=\Phi(S)
\end{array},
$$

Furthermore, if we repeat the arguments of preceding subsection and transform the Black-Scholes equation (88) into the heat equation, we obtain that $F(S, t)$ will be given by

$$
F(S, t)=\frac{1}{\sqrt{4 \pi \tau}} \int_{-\infty}^{\infty} \Phi\left(x^{\prime}\right) e^{-\left(x-x^{\prime}\right)^{2} / 4 \tau} d x^{\prime}
$$

where $\Phi(x)$ denotes the payoff function in terms of the dimensionless variable $x$; see (73). Expressing this result in terms of the original variables $S$ and $t$ yields a generalized Black-Scholes formula

$$
F(S, t)=\frac{e^{-r(T-t)}}{\sqrt{2 \pi \sigma^{2}(T-t)}} \int_{0}^{\infty} \Phi\left(S^{\prime}\right) e^{\left[\ln \left(\frac{S^{\prime}}{S}\right)-\left(r-\frac{1}{2} \sigma^{2}\right)(T-t)\right]^{2}} \frac{d S^{\prime}}{S^{\prime}}
$$

In summary, we have shown above that the BlackScholes model is complete. A market is said to be complete if every contingent claim can be replicated with a self-financing portfolio on the primary assets. Our "proof of completeness' given above is, of course, valid only for the case of European contingent claims with a simple payoff function $\Phi(S)$; it does not cover, for instance, pathdependent derivatives. It is possible however to give a formal proof that arbitrage-free models, such as the BlackScholes model, are indeed complete; see Sec. 5.3.

Comparing the generalized Black-Scholes formula (91) with the pdf of the geometric Brownian motion given in (54), we see that the former can be written in a convenient way as

$$
F(S, t)=e^{-r(T-t)} E_{t, S}^{Q}\left[\Phi\left(S_{T}\right)\right],
$$

where $E_{t, S}^{Q}[\cdot]$ denotes expectation value with respect to the probability density of a geometric Brownian motion with $\mu=r$, initial time $t$, final time $T$, and initial value $S$; see (54). In other words, the present value of a contingent claim can be computed simply as its discounted expected value at maturity, under an appropriate probability measure. This idea will become more clear after we discuss the notion of an equivalent martingale measure.

\section{Efficient markets: the martingale approach}

\subsection{Martingales}

The concept of a martingale plays a important rôle in finance [20]. Unfortunately, a proper introduction to martingales requires some knowledge of probability measure theory [21]. Here however we will give a rather intuitive discussion of martingales. For completeness we have listed in Appendix A some basic concepts from probability theory that would be required to make the following discussion more rigorous.

We begin by recalling that a probability space is a triple $(\Omega, \mathcal{F}, P)$, where
- $\Omega$ is the space of elementary events or outcomes $\omega$.

- $\mathcal{F}$ is a properly chosen family of subsets of $\Omega$, (i.e., a $\sigma$-algebra on $\Omega$ ).

- $P$ is a probability measure on $\mathcal{F}$.

In Finance, an outcome $\omega$ is a 'market situation.' The family of subsets $\mathcal{F}$ specifies the class of events to which probabilities can be assigned. This is done through the concept of a $\sigma$-algebra, whose formal definition is given in Appendix A. A probability measure $P$ on $\mathcal{F}$ is simply a function $P: \mathcal{F} \rightarrow[0,1]$, satisfying a few 'obvious requirements': $P(\emptyset)=0, P(\Omega)=1$, and $P\left(A_{1} \cup A_{2}\right)=$ $P\left(A_{1}\right)+P\left(A_{2}\right)$ if $A_{1} \cap A_{2}=\emptyset$. An element $A$ of $\mathcal{F}$, $A \in \mathcal{F}$, is called a "measurable set" or "observable event," meaning that it is possible to assign a "probability of occurrence," $P(A) \in[0,1]$, for this event. Hence, $\mathcal{F}$ is said to be the set of 'observable events.'

Suppose now that we have a random function $X$ : $\Omega \rightarrow \mathbf{R}$. If to every subset of $\Omega$ of the form $\{\omega: a \leq$ $X(\omega) \leq b\}$ there corresponds an event $A \subset \mathcal{F}$, then the function $X$ is said to be measurable with respect to $\mathcal{F}$ or simply $\mathcal{F}$-measurable. What this means is that it is possible to 'measure' (i.e., assign a probability to) events of the form $\{a \leq X \leq b\}$ through the obvious definition: $P(\{a \leq X \leq b\}) \equiv p(A)$. A $\mathcal{F}$-measurable function $X$ is called a random variable.

Let us next consider the notion of an "information flow." In a somewhat abstract way, we will represent the information available to an observer up to time $t$ as a $\sigma$-algebra $\mathcal{F}_{t} \subset \mathcal{F}$. In the context of Finance, the information set $\mathcal{F}_{t}$ would contain, for instance, the price history up to time $t$ of all assets in the economy. It is natural to assume that $\mathcal{F}_{s} \subset \mathcal{F}_{t}$ for $s \leq t$, since we expect that, as time goes on, we gain new information (and do not discard the old ones). Such a collection of $\sigma$-algebras represents an "information flow," or, more technically, a filtration.

Definition 5 A filtration or information flow is a collection $\left\{\mathcal{F}_{t}\right\}_{t \geq 0}$ of $\sigma$-algebras $\mathcal{F}_{t} \subset \mathcal{F}$ such that

$$
\mathcal{F}_{s} \subset \mathcal{F}_{t}, \quad \text { for } \quad 0 \leq s \leq t .
$$


Suppose now we have a stochastic process $X_{t}$ defined on $(\Omega, \mathcal{F}, P)$. (Think of $X_{t}$ as being, say, the price of a given stock.) If the values of $X_{t}$ can be completely determined from the information $\mathcal{F}_{t}$, for all $t \geq 0$, then the process $X_{t}$ is said to be adapted to the filtration $\left\{\mathcal{F}_{t}\right\}_{t \geq 0}$.

Definition 6 The process $X_{t}$ is adapted to the filtration $\left\{\mathcal{F}_{t}\right\}_{t \geq 0}$ if $X_{t}$ is $\mathcal{F}_{t}$-measurable for all $t \geq 0$.

A stochastic process $X_{t}$ naturally generates an information flow, denoted by $\mathcal{F}_{t}^{X}$, which represents the "information contained in the trajectories of $X(t)$ up to time $t$." A process $X(t)$ is obviously adapted to its natural filtration $\mathcal{F}_{t}^{X}$.

A last piece of mathematics is necessary to define a martingale, namely, the notion of conditional expectation. This appears in connection with the crucial question of how the information, $\mathcal{F}_{t_{0}}$, available at present time influences our knowledge about future values of $X(t)$. If $X(t)$ and $\mathcal{F}_{t_{0}}$ are not independent, then it is reasonable to expect that the information available up to the present time reduces the uncertainty about the future values of $X(t)$. To reflect this gain of information is useful to introduce a new stochastic process

$$
Z(t)=E\left[X_{t} \mid \mathcal{F}_{t_{0}}\right], \quad t>t_{0},
$$

where the symbol $E\left[X_{t} \mid \mathcal{F}_{t_{0}}\right]$ represents "the expected value of $X_{t}$, contingent on the information gathered up to time $t_{0}$."

A precise definition of conditional expectation is beyond the scope of these notes. Here it will suffice to say that given a random variable $Y$ on a probability space $(\Omega, \mathcal{F}, P)$ and another $\sigma$-algebra $\mathcal{F}^{\prime} \subset \mathcal{F}$, it is possible to define a random variable $Z=E\left[Y \mid \mathcal{F}^{\prime}\right]$, which represents "the expected value of $Y$, given the information contained in $\mathcal{F}^{\prime}$." The variable $Z$ is a coarser version of the original variable $Y$, in the sense that we have used the information on $\mathcal{F}^{\prime}$ to reduce the uncertainty about $Y$. The following two properties of conditional expectations will be necessary later:

$$
\begin{aligned}
& E\left[Y \mid \mathcal{F}_{t}\right]=Y, \quad \text { if } Y \text { is } \mathcal{F}_{t} \text {-measurable. } \\
& E\left[E\left[Y \mid \mathcal{F}_{t}\right]\right]=E[Y]
\end{aligned}
$$

The first property above is somewhat obvious: if $Y$ is $\mathcal{F}_{t^{-}}$ measurable then $Y$ and $\mathcal{F}_{t}$ 'contain the same information,' hence taking expectation of $Y$ conditional to $\mathcal{F}_{t}$ does not reduce the uncertainty about $Y$. The second property is the so-called law of iterated expectations, and basically represents the law of total probability.

After these mathematical preliminaries, we are now in a position to define martingales.

Definition 7 A stochastic process $M_{t}$ is called a martingale with respect to the filtration $\left\{\mathcal{F}_{t}\right\}_{t \geq 0}$ if

(i) $M_{t}$ is adapted to the filtration $\left\{\mathcal{F}_{t}\right\}_{t \geq 0}$

(ii) $E\left[\left|M_{t}\right|\right]<\infty$ for all $t \geq 0$

(iii) $E\left[M_{t} \mid \mathcal{F}_{t_{0}}\right]=M_{t_{0}}$ for all $t \geq t_{0}$
Condition (i) simply says that $M_{t}$ can be determined from the information available up to time $t$, whereas condition (ii) is a technicality. The defining property of a martingale is therefore condition (iii), which is usually referred to as the martingale condition. It says that the best prediction of future values of the process $M(t)$, contingent on the information available at the present time, is the current value $M_{t_{0}}$.

Because of property (iii), a martingale is usually described as a "fair game." To see why this is so, suppose that $M_{n}$ represents the fortune of a gambler at time $n$. (For convenience let us assume here that time is discrete.) The difference $h_{n}=M_{n}-M_{n-1}$ is then the amount the gambler wins on the $n$th play (a negative win is of course a loss). Now let us compute the gambler's expected gain on the $(n+1)$ th play, given the information up to time $n$ :

$$
\begin{aligned}
E\left[h_{n+1} \mid \mathcal{F}_{n}\right] & =E\left[M_{n+1}-M_{n} \mid \mathcal{F}_{n}\right] \\
& =E\left[M_{n+1} \mid \mathcal{F}_{n}\right]-E\left[M_{n} \mid \mathcal{F}_{n}\right] \\
& =M_{n}-M_{n} \\
& =0
\end{aligned}
$$

where in the third equality we used the martingale property and rule (93). We thus have that at each new play of the game the expected gain is null, and in this sense it is a "fair" game.

A Brownian motion $W(t)$ is a martingale with respect to its natural filtration. To show this, we only need to verify the martingale condition (since the other two conditions are trivially fulfilled):

$$
\begin{aligned}
E\left[W(t) \mid \mathcal{F}_{t_{0}}\right] & =E\left[W(t)-W\left(t_{0}\right)+W\left(t_{0}\right) \mid \mathcal{F}_{t_{0}}\right] \\
& =E\left[W(t)-W\left(t_{0}\right) \mid \mathcal{F}_{t_{0}}\right]+E\left[W\left(t_{0}\right) \mid \mathcal{F}_{t_{0}}\right] \\
& =0+W\left(t_{0}\right) \\
& =W\left(t_{0}\right) .
\end{aligned}
$$

In the third equality above we used the fact that the increments $W(t)-W\left(t_{0}\right)$ are independent of $\mathcal{F}_{t_{0}}$ and have zero mean, together with property (93). It is also possible to show that Itô stochastic integrals are martingales. Indeed, the theory of stochastic integration is intimately connected with martingale theory [5].

Another important property of martingales is that their expected value remains constant in time:

$$
E\left[M_{0}\right]=E\left[E\left[M_{t} \mid \mathcal{F}_{0}\right]\right]=E\left[M_{t}\right],
$$

where in first equality we used the martingale property, while in the second equality property (94) was used. Thus, a necessary (but not sufficient) condition for a process to be a martingale is that it have no drift. Therefore, a diffusion process of the form (40) is not a martingales unless the drift term vanishes. For this reason, the geometric Brownian motion (50) is not a martingale. It is possible, however, to introduce a new probability measure $Q$, with respect to which the geometric Brownian motion becomes a standard Brownian motion and hence a martingale, as discussed next. 


\subsection{Equivalent martingale measures}

Recall that a probability measure $P$ on a measurable space $(\Omega, \mathcal{F})$ is a function $P: \mathcal{F} \rightarrow[0,1]$ that assigns to every event $A \subset \mathcal{F}$ a real number $P(A) \in[0,1]$. Suppose now we have another probability measure $Q$ defined on the same space $(\Omega, \mathcal{F})$. We say that the probability measures $P$ and $Q$ are equivalent if the following condition is satisfied:

$$
Q(A)=0 \Longleftrightarrow P(A)=0, \quad \text { for all } \quad A \in \mathcal{F} .
$$

To get a better grasp on the meaning of the condition above, consider a random variable $X$ [on $(\Omega, \mathcal{F}, P)$ ]. If $Q$ is a probability measure equivalent to $P$, then condition (97) implies that there exists a function $\rho(X)$ such that expected values w.r.t $Q$ are calculated in the following way

$$
E_{Q}[g(X)]=E_{P}[\rho(X) g(X)],
$$

where $g(x)$ is an arbitrary function. Alternatively, we may write (98) in terms of probability densities:

$$
f_{Q}(x)=\rho(x) f_{P}(x),
$$

where $f_{P}(x)$ denotes the probability density of $X$ w.r.t the measure $P$ and $f_{Q}(x)$ is the density w.r.t $Q$. (The function $\rho(x)$ is called the Radon-Nikodym derivative of measure $Q$ with respect to measure $P$ [21].)

Consider now the Brownian motion with drift

$$
\tilde{W}(t)=a t+W(t), \quad 0 \leq t \leq T
$$

where $a$ is some constant. (The finite-horizon condition $t<T$ is a technicality that is not relevant for our purposes.) As already noted, $\tilde{W}(t)$ is not a martingale (since its expected value is not constant). However, there is an equivalent probability measure $Q$, with respect to which the process $\tilde{W}(t)$ becomes the standard Brownian motion (and hence a martingale). This result is known as Girsanov theorem.

Theorem 1 (Girsanov theorem) The process $\tilde{W}(t)$ given in (100) is a standard Brownian motion with respect to the probability measure $Q$ defined by

$$
f_{Q}(\tilde{x}, t)=M_{t}(\tilde{x}) f_{P}(\tilde{x}, t),
$$

where $M_{t}$ is the process

$$
M_{t}=\exp \left\{-a W_{t}-\frac{1}{2} a^{2} t\right\}=\exp \left\{-a \tilde{W}_{t}+\frac{1}{2} a^{2} t\right\} .
$$

Proof. For a formal proof see, e.g., [8]. Here we shall only sketch a proof of the fact that the process $\tilde{W}(t)$ is indeed distributed according to $\mathcal{N}(0, \sqrt{t})$, as the standard Brownian motion. First recall from (44) that the probability density $f_{P}(\tilde{x}, t)$ of $\tilde{W}(t)$ under the original measure $P$ is

$$
f_{P}(\tilde{x}, t)=\frac{1}{\sqrt{2 t}} \exp \left\{-\frac{(\tilde{x}-a t)^{2}}{2 t}\right\} .
$$

Now according to (101) and (102) we have that

$$
f_{Q}(\tilde{x}, t)=e^{-a \tilde{x}+\frac{1}{2} a^{2} t} f_{P}(\tilde{x}, t) .
$$

Inserting (103) into (104) then yields

$$
f_{Q}(\tilde{x}, t)=\frac{1}{\sqrt{2 t}} e^{-\tilde{x}^{2} / 2 t}
$$

which is precisely the pdf for the standard Brownian motion. Q.E.D.

One of the main applications of change of measures is to eliminate the drift in stochastic differential equations, so that with respect to the new measure $Q$ the process is a martingale. The measure $Q$ is then called an equivalent martingale measure. Constructing the equivalent martingale measure for an arbitrary SDE of the form (41) is a rather complicated procedure [5]. One important exception are linear SDE's where the Girsanov theorem gives the measure transformation in an explicit form, as shown below.

Consider the geometric Brownian motion discussed in Sec. 3.6. For technical reasons [8], let us restrict ourselves to its finite-horizon version:

$$
d S=\mu S d t+\sigma S d W, \quad t<T .
$$

where $\mu$ and $\sigma$ are positive constants. This equation can then be rewritten as

$$
d S=\sigma S\left(\frac{\mu}{\sigma} d t+d W\right)=\sigma S d \tilde{W}
$$

where

$$
\tilde{W}_{t}=(\mu / \sigma) t+W_{t}, \quad t<T .
$$

Now, according to Girsanov theorem, $\tilde{W}_{t}$ is a standard Brownian motion with respect to the measure $Q$ given in (101) with $a=\mu / \sigma$, and since the SDE (107) has no drift, its solution $S_{t}$ is a martingale w.r.t. the measure $Q$.

\subsection{The 'efficiency symmetry' and the no- arbitrage condition as its 'conservation law'}

The notion of an efficient market plays a crucial role in Finance. Roughly speaking, in an efficient market all relevant information is already reflected in the prices [3]. This means, in particular, that past prices give no additional information that is not already contained in the current price. In an efficient market prices thus adjust immediately to the arrival of new information. Since the content of future information and their effect on prices are unknown, it should be impossible to make definite predictions about future price based on the information available today. Thus, the best prediction for the expected future price (discounted to the present time) should be today's price. In other words, in a efficient market 'discounted prices' should be a martingale. This intuitive definition of efficiency is formalized below. [For technical reasons the results of this section will be 
restricted to the discrete-time case. Their extension to the continuous-time framework, although possible to a large extent, is more complicated and will not be attempted in the present notes; see, e.g., [5].]

Consider a market formed by two assets $(B, S)$, where $B$ is our usual risk-free free asset and $S$ is a risky asset. We suppose that $S(t)$ follows a stochastic process on a probability space $(\Omega, \mathcal{F}, P)$ endowed with a filtration $\left\{\mathcal{F}_{t}\right\}_{t \geq 0}$.

Definition 8 Suppose the market $(B, S)$ operates at discrete time $t_{n}, n=1,2, \ldots$ This market is said to be efficient if there exists (at least one) probability measure $Q$, equivalent to $P$, such that the 'discounted price' $\frac{S(t)}{B(t)}$ is a martingale with respect to the measure $Q$, that is,

$$
E^{Q}\left[\frac{S(t)}{B(t)} \mid \mathcal{F}_{t_{0}}\right]=\frac{S\left(t_{0}\right)}{B\left(t_{0}\right)}, \quad \text { for } \quad t_{0} \leq t
$$

where $E^{Q}$ means expected value w.r.t. the measure $Q$.

The requirement of efficiency, as defined above, is somewhat reminiscent of a symmetry principle in Physics. Indeed, we can recast definition (109) by saying that in a efficient market there exists a 'special measure Q' with respect to which discounted prices are invariant under a sort of 'time translation,' in the following sense:

$$
E^{Q}\left[\frac{S(t+T)}{B(t+T)} \mid \mathcal{F}_{t}\right]=\frac{S(t)}{B(t)}
$$

for any $T>0$.

In Physics, symmetry principles are intimately connected with conservation laws. (Recall, for instance, that the invariance of Newton's law under time translation implies conservation of energy.) It is thus only natural to ask whether the 'efficiency symmetry' above also leads to a 'conservation law.' Perhaps not surprisingly, this is indeed the case, as stated in the following theorem, which is sometimes referred to as the First Fundamental Theorem of asset pricing.

Theorem 2 Suppose the market $(B, S)$ operates at discrete time $t_{n}, n=1,2, \ldots$. Then this market is efficient if and only if it is arbitrage-free.

(See [5] for a proof.)

Recall that absence of arbitrage means that any selffinancing portfolio with zero initial value, and with no chance of becoming negative, will remain zero-valued for all subsequent times. More precisely, the no-arbitrage condition says that

$$
V(0)=0 \text { and } V(t) \geq 0 \text { a.s. } \Longrightarrow V(t)=0 \text { a.s. },
$$

where a.s. means almost surely, i.e., with probability 1 . The absence of arbitrage can thus be interpreted as a kind of "conservation law' for the "vacuum state" of the market: if you start in a state with zero initial money and do not take any risks, then you remain at this state for all times. I thus find it quite interesting that the so-called "no-free-lunch" condition can actually be seen as the conservation law associated with the efficiency symmetry.

Another very important result is the Second Fundamental Theorem of asset pricing, linking the completeness of a market to the uniqueness of its equivalent martingale measure.

Theorem 3 An arbitrage-free $(B, S)$-market is complete if and only if the equivalent martingale measure $Q$ is unique.

\section{(See [5] for a proof.)}

We already know that the Black-Scholes model is complete. Below we will calculate its equivalent martingale measure explicitly, and it will become clear from the construction that it is indeed unique.

\subsection{Pricing derivatives with the equivalent martingale measure}

The notion of an equivalent martingale measure can be used to price derivatives in a rather direct way, without having to solve a PDE. The idea is that in an efficient economy all financial assets are martingales with respect to the equivalent martingale measure $Q$. More precisely, if $F(S, t)$ is a contingent claim with maturity $T$ and payoff function $\Phi(S(T))$ then from (109) we have

$$
\frac{F(S, t)}{B(t)}=E_{t, S}^{Q}\left[\frac{\Phi(S(T))}{B(T)}\right]
$$

where the subscripts $t, S$ denote that the expected value is taken at present time $t$ and with current value $S$, i.e., conditional to the information available at time $t$. It is not hard to convince oneself that if the derivative price $F$ were not given by (110), then there would be an arbitrage opportunity.

In the Black-Scholes model, the risk-free asset is a bank account with fixed interest rate $r$, i.e., $B(t)=e^{r t}$, so that (112) becomes

$$
F(S, t)=e^{-r(T-t)} E_{t, S}^{Q}[\Phi(S(T))]
$$

or

$$
F(S, t)=e^{-r(T-t)} \int_{0}^{\infty} \Phi\left(S^{\prime}\right) f_{Q}\left(S^{\prime}, T ; S, t\right) d S^{\prime},
$$

where $f_{Q}\left(S, t ; S_{0}, t_{0}\right)$ denotes the probability density, under the equivalent martingale measure $Q$, of the process $S(t)$ with initial value $S\left(t_{0}\right)=S_{0}$. All that remains to be done now is to find the equivalent martingale measure $Q$ for the Black-Scholes model. To do this, consider the process

$$
Z(t)=\frac{S(t)}{B(t)}=e^{-r t} S(t)
$$


We then have

$$
\begin{aligned}
d Z & =-r e^{-r t} S d t+e^{-r t} d S \\
& =(\mu-r) Z d t+\sigma Z d W \\
& =\sigma Z d \tilde{W},
\end{aligned}
$$

where

$$
\tilde{W}(t)=[(\mu-r) / \sigma] t+W(t) .
$$

Now recall that in the Black-Scholes model the stock price follows a geometric Brownian motion

$$
d S=\mu S d t+\sigma S d W
$$

which in terms of the process $\tilde{W}(t)$ given in (116) reads

$$
d S=r S d t+\sigma S d \tilde{W}
$$

From Girsanov theorem we know that there is a equivalent martingale measure $Q$ that turns $\tilde{W}(t)$ into a Brownian motion. Equation (118) then shows that w.r.t the measure $Q$ the price $S(t)$ follows a geometric Brownian motion with mean rate of return equal to $r$. The probability density $f_{Q}\left(S^{\prime}, T ; S, t\right)$ can now be obtained directly from (54), by simply setting $\mu=r$ and $S_{0}=S$. One then gets

$$
f_{Q}\left(S^{\prime}, T ; S, t\right)=\frac{1}{S^{\prime} \sqrt{2 \sigma^{2} \tau}} \exp \left\{-\frac{\left[\ln \left(\frac{S^{\prime}}{S}\right)-\left(r-\frac{1}{2} \sigma^{2}\right) \tau\right]^{2}}{2 \sigma^{2} \tau}\right\},
$$

where $\tau=T-t$. Inserting (119) into (114) we obtain

$$
F(t, S)=\frac{e^{-r(T-t)}}{\sqrt{2 \pi \sigma^{2}(T-t)}} \int_{0}^{\infty} \Phi\left(S^{\prime}\right) e^{\left[\ln \left(S^{\prime} / S\right)-\left(r-\frac{1}{2} \sigma^{2}\right)(T-t)\right]^{2}} \frac{d S^{\prime}}{S^{\prime}},
$$

which is precisely the expression obtained for the generic solution of the Black-Scholes equation given in (92). In the case of a European call option we have $\Phi\left(S^{\prime}\right)=\max \left(S^{\prime}-\right.$ $K, 0)$, which inserted into (120) yields, after some algebra, the Black-Scholes formula (84).

It is interesting to notice that under the equivalent martingale measure $Q$ the stock price in the Black-Scholes model follows a geometric Brownian motion with the mean rate of return equal to the risk-free interest rate $r$; see (118). It is as if all investors were risk neutral, in the sense they would be willing to invest on a risky stock even though its expected return is just what a risk-free bank account would yield. For this reason, the pricing method based on the equivalent martingale measure is commonly referred to as risk neutral valuation. Of course, actual investors are not risk neutral. However, in an efficient market there is a 'special reference frame' where investors can be treated as if they were indeed insensitive to risk.

\section{Beyond the Standard Model of Fi- nance I: Non-Gaussian Distribu- tions}

We have seen above that the Black-Scholes model is an elegant and powerful theoretical construct: it is complete, efficient, arbitrage-free, and Gaussian (in the sense that the stock returns are normally distributed). It is thus important to ask whether real markets actually fit into this nice framework.
There are two main ways in which real markets may deviate from the standard Black-Scholes model: i) the returns may not be normally distributed or ii) there may exist longmemory effects on the time series. In this Section we will discuss the possibility that asset prices may follow a nonGaussian stable Lévy process, while in the next section we investigate whether financial data might exhibit long-term memory.

Mandelbrot [22] in 1963 was perhaps the first person to challenge the paradigm that returns are normally distributed. He analyzed cotton prices on various exchanges in the United States and found evidences that their distribution of returns decays as a power law and hence much slower than a Gaussian. An example of this 'fat tail' behavior can be seen in Fig. 8, where I plot the distribution for the returns of the Ibovespa index. In this figure, it is also shown a Gaussian distribution with the variance of the data, which appears as a parabola in the linear-log scale of the graph. One clearly sees that the empirical distribution does indeed have 'fatter tails' when compared with the Gaussian distribution.

The Gaussian distribution is special for two main reasons. The first one is the Central Limit Theorem [21] that states that the sum of infinitely many independent random variables (with finite variance) will converge to a Gaussian variable. The second one is the fact that it is a stable distribution, in the sense that the sum of two independent Gaussian random variables is also a Gaussian variable. It is thus natural to ask whether there are other stable distributions. The French mathematician Paul Lévy showed that there is indeed a whole family of stable distributions of which the Gaussian is but one particular case. In what follows, I will 
first introduce the so-called Lévy stable distributions and then briefly discuss their possible applications to financial data.

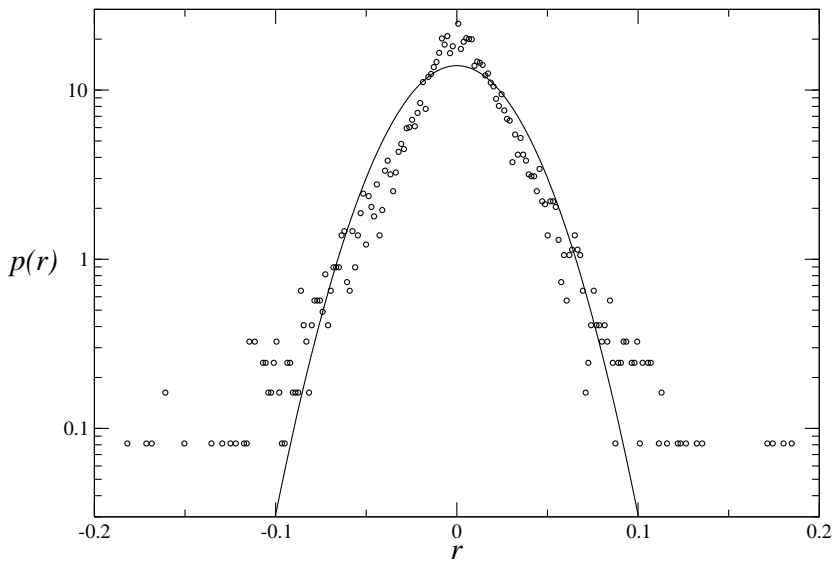

Figure 8. Distribution of the daily Ibovespa returns (open circles). The solid line correspond to a Gaussian distribution with the same variance as that of the empirical distribution.

\subsection{The stable Lévy distributions}

Let $X$ be a random variable with probability density function (pdf) given by $p(x)$. We recall that the characteristic function $\varphi(z)$ of a random variable $X$ is the Fourier transform of its pdf $p(x)$ :

$$
\varphi(z)=\int_{-\infty}^{\infty} p(x) e^{i z x} d x .
$$

Now let $X_{1}$ and $X_{2}$ be two independent random variables with pdf's $p_{1}\left(x_{1}\right)$ and $p_{2}\left(x_{2}\right)$. Since $X_{1}$ and $X_{2}$ are independent, the pdf of the sum $X=X_{1}+X_{2}$ is the convolution of the original pdf's:

$$
p(x)=\int_{-\infty}^{\infty} p_{1}(s) p_{2}(x-s) d s
$$

Let now $\varphi(z, 2)$ denote the characteristic function of $X$. In view of the convolution theorem which states that the Fourier transform of the convolution is the product of the Fourier transforms, it then follows that

$$
\varphi(z, 2)=\varphi_{1}(z) \varphi_{2}(z) .
$$

Suppose furthermore that $X_{1}$ and $X_{2}$ are identically distributed, that is,

$$
X_{1} \stackrel{\mathrm{d}}{=} X_{2}
$$

or alternatively

$$
\varphi_{1}(z)=\varphi_{2}(z)=\varphi(z)
$$

(Recall that the symbol $\stackrel{\mathrm{d}}{=}$ denotes equality in the distribution sense.) From (122) and (124) it then follows that

$$
\varphi(z, 2)=[\varphi(z)]^{2} .
$$

In general, if $X=\sum_{i=1}^{N} X_{i}$, where the $X_{i}$ 's are independent and identically distributed (i.i.d.) random variables, then

$$
\varphi(z, N)=[\varphi(z)]^{N},
$$

from which the pdf of $X$ can be obtained by calculating the inverse Fourier transform. Note that the pdf of the sum of $N$ i.i.d. random variables will in general be quite different from the pdf of the individual variables. There is however a special class of distribution, the stable distributions, for which the pdf of the sum has the same functional form of the individual pdf's.

Definition 9 A probability distribution $p(x)$ is stable if for each $N \geq 2$ there exist numbers $a_{N}>0$ and $b_{N}$, such that, if $X_{1}, \ldots, X_{N}$ are i.i.d. random variables with distribution $p(x)$, then

$$
X_{1}+\ldots+X_{N} \stackrel{d}{=} a_{n} X_{i}+b_{N} .
$$

In other words, a distribution is stable if its form is invariant under addition, up to a rescaling of the variable by a translation and a dilation. More precisely, if $p(x, N)$ denotes the probability density of $X=\sum_{i=1}^{N} X_{i}$, where the $X_{i}$ 's are i.i.d. variables with a stable distribution $p(x)$, then (126) implies that

$$
p(x, N)=\frac{1}{a_{N}} p\left(\frac{x-b_{N}}{a_{N}}\right) .
$$

Stability of probability distributions has a nice interpretation from an economic standpoint. To see this, suppose that the variables $X_{i}$ represent daily increments of a given financial asset. Stability then means preservation of the distribution under time aggregation, which is a rather natural property to expect from financial data.

As already mentioned, the Gaussian distribution is stable. To see this, recall that the Fourier transform of a Gaussian is a Gaussian and that the product of Gaussians is again a Gaussian, so that from (125) it follows that the characteristic function $\varphi(z, N)$ will indeed be that of a Gaussian variable. More precisely, we have that the characteristic function of a normal variable $\mathcal{N}(0, \sigma)$ is $\varphi(z)=e^{-\left(\sigma^{2} / 2\right) z^{2}}$, which inserted into (125) immediately yields $\varphi(z, N)=$ $e^{-\left(N \sigma^{2} / 2\right) z^{2}}$. Thus, the sum of $N$ i.i.d. normal variables is normally distributed with standard deviation $\sqrt{N} \sigma$, that is, $X \stackrel{d}{=} \mathcal{N}(0, \sqrt{N} \sigma)$, which in turn implies that

$$
p(x, N)=\frac{1}{\sqrt{N}} p\left(\frac{x}{\sqrt{N}}\right) .
$$

Thus, in the case of the Gaussian distribution we have $a_{N}=$ $1 / \sqrt{N}$ and $b_{N}=0$.

The class of stable distributions is rather small and was completely determined by the mathematicians P. Lévy and A. Ya. Khintchine in the 1920's. Here we shall restrict ourselves to the subclass of symmetric distribution. In this case, the characteristic function is given by

$$
\varphi_{\alpha}(z)=e^{-a|z|^{\alpha}}
$$

where $0<\alpha \leq 2$ and $a>0$. The parameter $\alpha$ is called the stability exponent and $a$ is a scale factor. Taking the inverse Fourier transform of $\varphi_{\alpha}(z)$ we obtain the corresponding pdf $p_{\alpha}(x)$ : 


$$
p_{\alpha}(x)=\frac{1}{2 \pi} \int_{-\infty}^{\infty} \varphi_{\alpha}(z) e^{-i z x} d z=\frac{1}{\pi} \int_{0}^{\infty} e^{-a z^{\alpha}} \cos (z x) d z .
$$

Unfortunately, however, only for two particular values of $\alpha$ can the integral above be explicitly calculated:

- $\alpha=1$ (Lorentzian or Cauchy distribution):

$$
p(x)=\frac{2 a}{\pi} \frac{1}{x^{2}+4 a^{2}} .
$$

- $\alpha=2$ (Gaussian distribution):

$$
p(x)=\frac{1}{8 \pi a} e^{-x^{2} / 4 a} .
$$

Note also that Lévy distributions are not defined for $\alpha>2$, because in this case the function obtained from (130) is not everywhere positive.

Although, for arbitrary $\alpha$ the pdf $p_{\alpha}(x)$ cannot be found in closed form, its asymptotic behavior for large $x$ can be easily calculated from (130). Here one finds [5] that

$$
p_{\alpha}(x) \approx \frac{C_{\alpha}}{|x|^{1+\alpha}}, \quad|x| \rightarrow \infty,
$$

where

$$
C_{\alpha}=\frac{a}{\pi} \Gamma(1+\alpha) \sin \frac{\pi \alpha}{2} .
$$

We thus see that the Lévy distribution with $\alpha<2$ has the interesting property that it shows scaling behavior for large $x$, i.e., $p(x)$ decays as a power-law.

Another important scaling relation for the Lévy distribution can be obtained, as follows. First note that for symmetric stable distribution we necessarily have $b_{N}=0$. Now using (125) and (129), we easily find that the dilation factor $a_{N}$ in (127) is

$$
a_{N}=N^{1 / \alpha}
$$

so that (127) becomes

$$
p_{\alpha}(x, N)=\frac{p_{\alpha}\left(N^{1 / \alpha} x\right)}{N^{1 / \alpha}},
$$

which implies that

$$
p(0, N)=\frac{p(0)}{N^{1 / \alpha}}
$$

One can then use this scaling relation to estimate the index $\alpha$ of the Lévy distribution: in a log-log plot of $p(0, N)$ against $N$, the slope of a linear fit gives precisely $1 / \alpha$; see Sec. 6.3 below.

The power-law decay of Lévy distributions implies, of course, the absence of a characteristic scale. The downside of this is that all Lévy distributions have infinite variance! In fact, all moments of order higher than 1 are infinite, since $E\left[|x|^{n}\right]$ diverges for $n \geq \alpha$, as can be readily shown from (131). Processes with infinite variance are not physically plausible, so several prescriptions to truncate the Lévy distribution at some large scale has been proposed, as discussed next.

\subsection{Truncated Lévy distributions}

To circumvent the problem of infinite variance in Lévy distributions, several truncation prescriptions have been proposed in the literature. In a general they can be written as

$$
p(x)=p_{\alpha}(x) \Phi(x),
$$

where $\Phi(x)$ is a cut-off function to be chosen in such way that the variance of the truncated distribution is finite. For example, two possible choices that have been used to model the distributions of financial asset prices are given below

- Abruptly truncated Lévy distribution (ATLD):

$$
\Phi(x)=\Theta\left(x_{c}-|x|\right),
$$

where $\Theta(x)$ is the Heaviside function and $x_{c}$ is some cut-off length scale.

- Exponentially truncated Lévy distribution (ETLD):

$$
\Phi(x)=A e^{-\lambda|x|},
$$

where $\lambda>0$ and $A$ is a normalization factor.

Other variants of truncated Lévy distributions that have also been considered in the literature are the gradually truncated Lévy distribution [23] and the exponentially damped Lévy distributions [24].

Since a truncated Lévy distribution has finite variance, then by the central limit theorem the distribution of the sum $X=X_{1}+\ldots+X_{N}$ of $N$ i.i.d variables with such a distribution will converge to a Gaussian distribution for large $N$. However, this convergence is usually very slow-for financial data it is typically in the order of tens of days; see below. For shorter time scales, non-Gaussian behavior may thus be of practical relevance.

\subsection{Lévy distributions in Finance}

Lévy distribution have been used, for example, by Mantegna \& Stanley [9] to model the distribution of changes in the stock index S\&P500 of the American Stock Exchange. They analyzed high-frequency data (one-minute quotes of the S\&P500) over the period from January 1984 to December 1989. From the original time series $Y(t)$ of the index values, they first generated time series corresponding to index changes during intervals of $N$ minutes:

$$
Z_{N}(t) \equiv Y(t+N)-Y(t)
$$


They then computed the empirical pdf $p(z, N)$ and analyzed the scaling of $p(0, N)$ with $N$. In a log-log plot $p(0, N)$ showed a linear behavior, as predicted by (135), with a slope corresponding to $\alpha=1.4$ for $30<N<1000$ minutes [9]. For $N>10^{4}$ the slope of $p(0, N)$ approaches -0.5 , indicating convergence to a Gaussian behavior. In the Lévy regime (i.e., small $N$ ), however, the tail of their empirical pdf decays slower than Gaussian but faster than a pure Lévy distribution with the exponent $\alpha$ found from above scaling argument. These facts thus suggest that a truncated Lévy distribution would perhaps be more appropriate for modeling the actual distribution. Indeed, Bouchaud and Potters [10] found that the probability of 15-minute changes of the S\&P500 index is well described by a ETLD with $\alpha=1.5$.

The ETLD has also been applied by Miranda \& Riera [25] to study the daily returns of Ibovespa index of the São Paulo Stock Exchange in the period 1986-2000. From the daily closing values $Y(t)$ of the Ibovespa, they first calculated the time series for the returns in intervals of $N$ days

$$
r_{N}(t)=\log Y(t+N)-\log Y(t)
$$

and then computed the corresponding pdf's for $p(r, N)$. From the scaling of $p(0, N)$ they found $\alpha \simeq 1.6-1.7$ for $N<20$ days, whereas for larger $N$ a Gaussian-like behavior (i.e., $\alpha=0.5$ ) was observed.

Many other applications of Lévy processes in Finance have been discussed in the literature [26]. For example, a model for option pricing has recently been considered where the price of the underlying asset is assumed to follow a truncated Lévy process [27]. More recently, there have accumulated evidences $[28,29,30]$ that in certain cases financial data may be better described by exponential distributions, rather than by Lévy or Gaussian distributions.

\section{Beyond the Standard Model of Fi- nance II: Long-Range Correlations}

In this section, we discuss the possibility that asset prices might exhibit long-range correlations and thus may need to be described in terms of long-memory processes, such as the fractional Brownian motion.

\subsection{Fractional Brownian motion}

The fractional Brownian motion (FBM) is a Gaussian process $\left\{W_{H}(t), t>0\right\}$ with zero mean and stationary increments, whose variance and covariance are given by

$$
\begin{gathered}
E\left[W_{H}^{2}(t)\right]=t^{2 H}, \\
E\left[W_{H}(s) W_{H}(t)\right]=\frac{1}{2}\left(s^{2 H}+t^{2 H}-|t-s|^{2 H}\right),
\end{gathered}
$$

where $0<H<1$. The FBM $W_{H}(t)$ is a self-similar process, in the sense that

$$
W_{H}(a t) \stackrel{d}{=} a^{H} W_{H}(t)
$$

for all $a>0$. A sample path of a FBM is therefore a fractal curve with fractal dimension $D=1 / H$. The parameter $H$ is called the self-similarity exponent or the Hurst exponent. For $H=1 / 2$ the process $W_{H}(t)$ corresponds to the usual Brownian motion, in which case the increments $X_{t}=W_{H}(t+1)-W_{H}(t)$ are statistically independent, corresponding to white noise. On the other hand, for $H \neq 1 / 2$ the increments $X_{t}$, known as fractional white noise, display long-range correlation in the sense that

$$
E\left[X_{t+h} X_{t}\right] \simeq 2 H(2 H-1) h^{2 H-2} \quad \text { for } \quad h \rightarrow \infty,
$$

as one can easily verify from (139) and (140). Thus, if $1 / 2<H<1$ the increments of the FBM are positively correlated and we say that the process $W_{H}(t)$ exhibits persistence. Likewise, for $0<H<1 / 2$ the increments are negatively correlated and the FBM is said to show antipersistence. Sample FBM paths with $H=0.2,0.5$, and 0.8 are shown in Fig. 9.

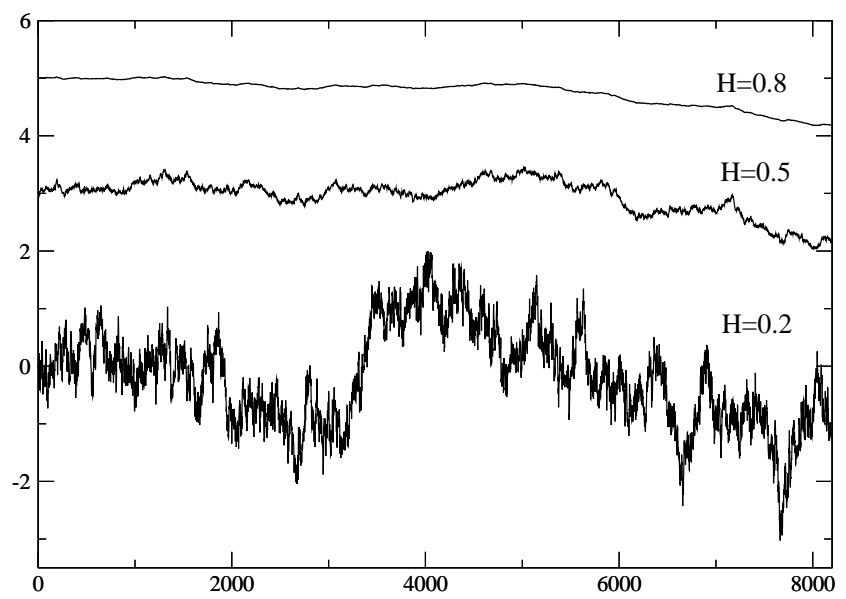

Figure 9. Sample paths of fractional Brownian motion.

Several estimators for the exponent $H$ have been discussed in the literature; see, e.g., Ref. [31] for a comparison among some of them. One general methodology consists in estimating how the 'amount of fluctuation' within a time window of size $\tau$ scales with $\tau$. Specific methods, such as the Hurst rescaled range (R/S) analysis [32] or the Detrendend Fluctuation Analysis [33, 34], differ basically on the choice of the fluctuation measure. Here I shall discuss only the DFA that has proven to be a more reliable estimator for $H$ than the Hurst R/S analysis [35].

\subsection{Detrended fluctuation analysis}

Suppose we have a time series $r(t), t=1, \ldots, T$, corresponding to, say, daily returns of a financial asset. To implement the DFA, we first integrate the original time series $r(t)$ to obtain the cumulative time series $X(t)$ :

$$
X(t)=\sum_{t^{\prime}=1}^{t}\left(r\left(t^{\prime}\right)-\bar{r}\right), \quad t=1, \ldots, T,
$$


where

$$
\bar{r}=\frac{1}{T} \sum_{t^{\prime}=1}^{T} r\left(t^{\prime}\right) .
$$

Next we break up $X(t)$ into $N$ non-overlapping time intervals, $I_{n}$, of equal size $\tau$, where $n=0,1, \ldots, N-1$ and $N$ corresponds to the integer part of $T / \tau$. We then introduce the local trend function $Y_{\tau}(t)$ defined by

$$
Y_{\tau}(t)=a_{n}+b_{n} t \quad \text { for } \quad t \in I_{n},
$$

where the coefficients $a_{n}$ and $b_{n}$ represent the least-square linear fit of $X(t)$ in the interval $I_{n}$. Finally, we compute the rescaled fluctuation function $F(\tau)$ defined as [35]

$$
F(\tau)=\frac{1}{S} \sqrt{\frac{1}{n \tau} \sum_{t=1}^{N \tau}\left[X(t)-Y_{\tau}(t)\right]^{2}},
$$

where $S$ is the data standard deviation

$$
S=\sqrt{\frac{1}{T} \sum_{t=1}^{T}\left(r_{t}-\bar{r}\right)^{2}}
$$

The Hurst exponent $H$ is then obtained from the scaling behavior of $F(\tau)$ :

$$
F(\tau)=C \tau^{H}
$$

where $C$ is a constant independent of the time lag $\tau$.

In a double-logarithmic plot the relationship (148) yields a straight line whose slope is precisely the exponent $H$, and so a linear regression of the empirical $F(\tau)$ will immediately give $H$. One practical problem with this method, however, is that the values obtained for $H$ are somewhat dependent on the choice of the interval within which to perform the linear fit $[35,36]$. It is possible to avoid part of this difficulty by relying on the fact that for the fractional Brownian motion, the fluctuation function $F(\tau)$ can be computed exactly [31]:

$$
F_{H}(\tau)=C_{H} \tau^{H}
$$

where

$$
C_{H}=\left[\frac{2}{2 H+1}+\frac{1}{H+2}-\frac{2}{H+1}\right]^{1 / 2} .
$$

In (149) we have added a subscript $H$ to the function $F$ to denote explicitly that it refers to $W_{H}(t)$. Equation (149) with (150) now gives a one-parameter estimator for the exponent $H$ : one has simply to adjust $H$ so as to obtain the best agreement between the theoretical curve predicted by $F_{H}(\tau)$ and the empirical data for $F(\tau)$.

\subsection{Fractional Brownian motion in Finance}

The idea of using the FMB for modeling asset price dynamics dates back to the work of Mandelbrot \& van Ness [37]. Since then, the Hurst exponent has been calculated (using different estimators) for many financial time series, such as stock prices, stock indexes and currency exchange rates [38, 39, 40, 41, 35]. In many cases [38] an exponent
$H>1 / 2$ has been found, indicating the existence of longrange correlation (persistence) in the data. It is to be noted, however, that the values of $H$ computed using the traditional $\mathrm{R} / \mathrm{S}$-analysis, such as those quoted in [38], should be viewed with some caution, for this method has been shown [35] to overestimate the value of $H$. In this sense, the DFA appears to give a more reliable estimates for $H$.

An example of the DFA applied to the returns of the Ibovespa stock index is shown in Fig. 10 (upper curve). In this figure the upper straight line corresponds to the theoretical curve $F_{H}(\tau)$ given in (149) with $H=0.6$, and one sees an excellent agreement with the empirical data up to $\tau \simeq 130$ days. The fact that $H>0.5$ thus indicates persistence in the Ibovespa returns. For $\tau>130$ the data deviate from the initial scaling behavior and cross over to a regime with a slope closer to $1 / 2$, meaning that the Ibovespa looses its 'memory' after a period of about 6 months. Also shown in Fig. 10 is the corresponding $F(\tau)$ calculated for the shuffled Ibovespa returns. In this case we obtain an almost perfect scaling with $H=1 / 2$, as expected, since the shuffling procedure tends to destroys any previously existing correlation.

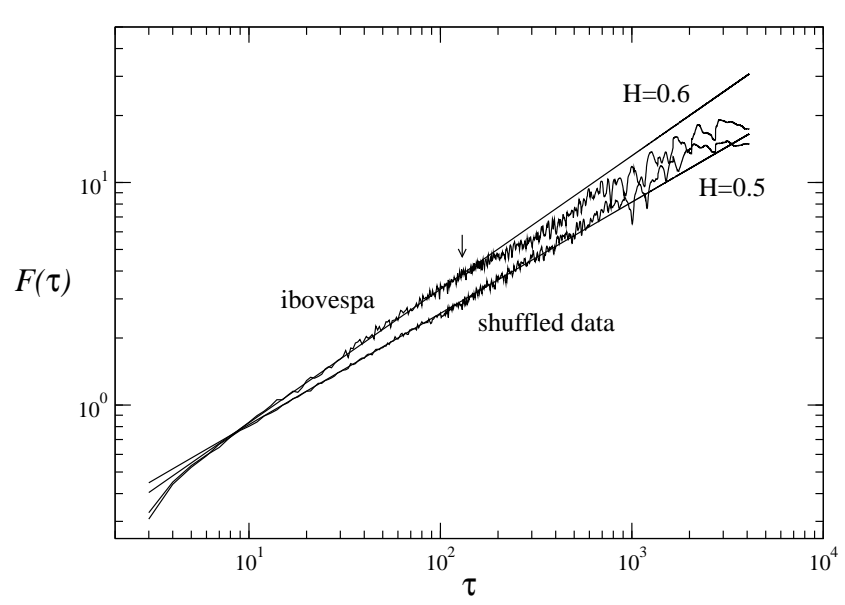

Figure 10. Fluctuation function $F(\tau)$ as a function of $\tau$ for the returns of the Ibovespa index (upper curve) and for the shuffled data (lower curve). The upper (lower) straight line gives the theoretical curve $F_{H}(\tau)$ for $H=0.6(H=1 / 2)$.

As already mentioned, the Hurst exponent has been calculated for many financial time series. In the case of stock indexes, the following interesting picture appears to be emerging from recent studies [40, 41, 35]: large and more developed markets, such as the New York and the London Stock Exchanges, usually have $H$ equal to (or slightly less than) $1 / 2$, whereas less developed markets show a tendency to have $H>1 / 2$. In other words, large markets seem indeed to be 'efficient' in the sense that $H \simeq 1 / 2$, whereas less developed markets tend to exhibit long-range correlation. A possible interpretation for this finding is that smaller markets are conceivably more prone to "correlated fluctuations' and perhaps more susceptible to being pushed around by aggressive investors, which may explain in part a Hurst exponent greater than $1 / 2$. 
It should also be pointed out that a considerable timevariability of the exponent $H$ for stock indexes has been found $[41,35]$, indicating that the data in such cases cannot be modeled in terms of stationary stochastic processes. (A time-varying $H$ has also been observed in other financial data, such as, currency exchange rate [39].) In such cases, the stationarity assumption is only a rather crude approximation [35]. Furthermore, the time dependence of the Hurst exponent is an indication that the underlying process might be multifractal rather than monofractal; see below.

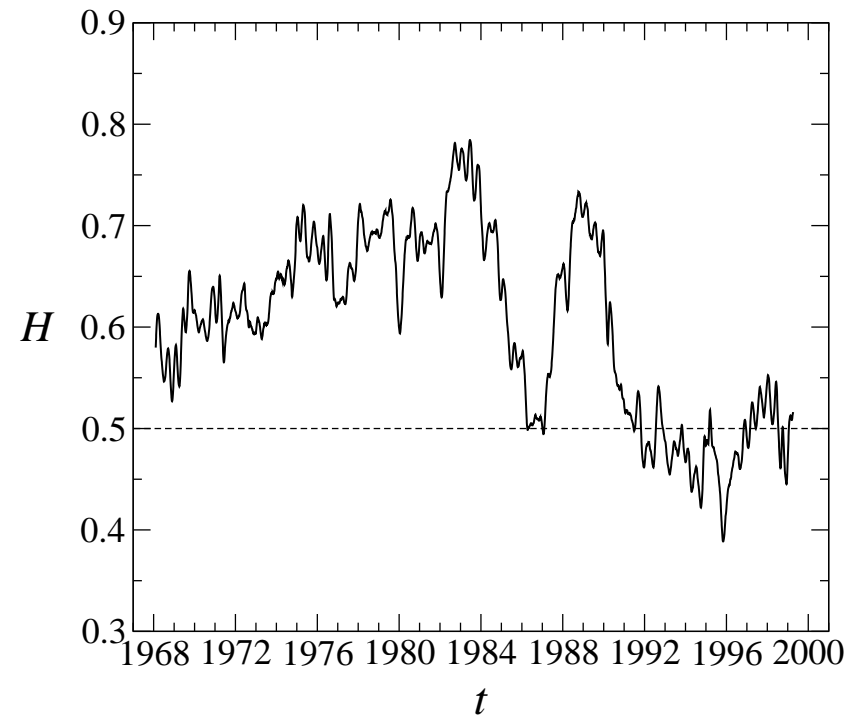

Figure 11. The Hurst exponent $H$ for the Ibovespa as a function of time. Here $H$ was computed in two-year time intervals, with the variable $t$ denoting the origin of each such interval.

A time-varying Hurst exponent has been observed for the Brazilian stock market. This case is of particular interest because in the recent past Brazil was plagued by runaway inflation and endured several ill-fated economic plans designed to control it. To analyze the effect of inflation and the economic plans, Costa and Vasconcelos [35] calculated a time-varying Hurst exponent for the Ibovespa returns, computed in three-year time windows for the period 1968-2001. A similar analysis but with two-year time windows is shown in Fig. 11. One sees from this figure that during the 1970's and 1980's the curve $H(t)$ stays well above $1 / 2$, the only exception to this trend occurring around the year 1986 when $H$ dips momentarily towards $1 / 2$ - an effect caused by the launch of the Cruzado economic Plan in February 1986 [35]. In the early 1990's, after the launching of the Collor Plan, we observe a dramatic decline in the curve $H(t)$ towards $1 / 2$, after which it remained (within some fluctuation) around $1 / 2$. This fact has led Costa and Vasconcelos [35] to conclude that the opening and consequent modernization of the Brazilian economy that begun with the Collor Plan resulted in a more efficient stock market, in the sense that $H \simeq 0.5$ after 1990. In Fig. 11, one clearly sees that after the launching of a new major economic plan, such as the Cruzado Plan in 1986 and the Collor Plan in 1990, the Hurst exponent decreases. This effect has, of course, a simple economic inter- pretation: a Government intervention on the market is usually designed to introduce "anti-persistent effects," which in turn leads to a momentary reduction of $H$. Note also that only after 1990 does the curves $H(t)$ goes below 1/2. This finding confirms the scenario described above that more developed markets (as Brazil became after 1990) tend to have $H \lesssim 1 / 2$.

\subsection{Option pricing under the FBM assump- tion}

We have seen above that often times asset prices have a Hurst exponent different from $1 / 2$. In such cases, the standard Black-Scholes model does not apply, since it assumes that returns follow a Brownian motion $(H=0.5)$. A more appropriate model for the return dynamics would be the fractional Brownian motion (FBM). Indeed, a 'fractional Black-Scholes model' has been formulated, in which it is assumed that the stock price $S$ follows a geometric fractional Brownian motion given by

$$
d S=\mu S d t+\sigma S d W_{H},
$$

where $W_{H}(t)$ is the standard FBM. The fractional stochastic differential equation above is shorthand for the integral equation

$$
S(t)=S(0)+\mu \int_{0}^{t} S\left(t^{\prime}\right) d t^{\prime}+\sigma \int_{0}^{t} S\left(t^{\prime}\right) d W_{H}\left(t^{\prime}\right) .
$$

To make mathematical sense of this equation is, of course, necessary to define stochastic integrals with respect to $W_{H}(t)$. Here it suffices to say that a fractional Itô calculus can indeed be rigorously defined [42] that shares (in an appropriate sense) many of the properties of the usual Itô calculus. In the context of this fractional Itô calculus is possible to prove that the solution to (151) is given by

$$
S(t)=S(0) \exp \left\{\mu t-\frac{1}{2} \sigma^{2} t^{2 H}+\sigma W_{H}(t)\right\} .
$$

Compare this expression with (53).

One can show [42] that the fractional Black-Scholes model is complete and arbitrage-free. To price derivatives with this model, one can apply the same $\Delta$-hedging argument used before, which now leads to the 'fractional BlackScholes equation.' The result is summarized in the following theorem.

Theorem 4 In the fractional Black-Scholes model, the price $F(S, t)$ of a European contingent claim with payoff $\Phi(S(T))$ is given by the solution to the following boundaryvalue problem

$$
\begin{array}{r}
\frac{\partial F}{\partial t}+H \sigma^{2} t^{2 H-2} S^{2} \frac{\partial^{2} F}{\partial S^{2}}+r S \frac{\partial F}{\partial S}-r F=0 \\
F(T, S)=\Phi(S) .
\end{array}
$$


[Compare with (88).]

For the case of a European call option the solution to the problem above can be found in closed form. Alternatively, one can obtain the option pricing formula directly from the equivalent martingale measure, without having to solve the above PDE. The final result for this 'fractional Black-Scholes formula' is given below [43].

Theorem 5 In the fractional Black-Scholes model, the price of a European call option with strike price $K$ and maturity $T$ is given by

$$
C(S, t)=S N\left(d_{1}\right)-K e^{-r(T-t)} N\left(d_{2}\right),
$$

where

$$
\begin{aligned}
& d_{1}=\frac{\ln \left(\frac{S}{K}\right)+r(T-t)+\frac{1}{2} \sigma^{2}\left(T^{2 H}-t^{2 H}\right)}{\sigma \sqrt{T^{2 H}-t^{2 H}}} \\
& d_{2}=\frac{\ln \left(\frac{S}{K}\right)+r(T-t)-\frac{1}{2} \sigma^{2}\left(T^{2 H}-t^{2 H}\right)}{\sigma \sqrt{T^{2 H}-t^{2 H}}}(
\end{aligned}
$$

[Compare with (84).]

The fractional Black-Scholes formula has been applied to price some options traded on the Brazilian market [44]. Here, however, the option prices obtained with the formula above resulted considerably higher than those from the usual Black-Scholes formula. The practical relevance of the fractional Black-Scholes model to real markets thus needs to be investigated further.

\subsection{Multifractality in Finance}

The fact that the Hurst exponents of financial data often display considerable variability in time indicates, as already mentioned, that such time series cannot be satisfactorily modeled in terms of a fractional Brownian motion, which is characterized by a constant $H$ and would thus capture only a sort of average behavior of the actual price dynamics [35]. In such cases, it would be more appropriate to model the data as a multifractal process.

The notion of a multifractal was first introduced in the context of dynamical systems to describe physical processes taking place on a fractal support [45]. A rigorous exposition of multifractal measures is beyond the scope of the present notes, and so we will content ourselves with a rather intuitive description of multifractality. The basic idea here is that a monofractal process, such as the FBM, is characterized by a single exponent $H$, whereas for a multifractal a whole family (spectrum) of exponents is necessary, one for each moment of the distribution.

We have seen above that the FBM is a Gaussian process whose standard deviation scales with time as

$$
\sqrt{E\left[W_{H}^{2}(t)\right]}=t^{H} .
$$

If we now introduce the generalized Hurst exponents $H_{q}$ as the corresponding scaling exponent for the $2 q$-th moment, that is,

$$
\left\{E\left[W_{H}^{2 q}(t)\right]\right\}^{1 / 2 q}=C_{q} t^{H_{q}}
$$

where $C_{q}$ is a constant, it then immediately follows from property (19) of the Gaussian distribution that

$$
H_{q}=H
$$

That is, all higher-order Hurst exponents of the FBM are equal to $H$ itself and hence the FBM is said to be a monofractal. Our working definition of a multifractal will then be a process for which the generalized Hurst exponents $H_{q}$ vary with $q$, or alternatively, that the quantity $q H_{q}$ does not scale linearly with $q$.

In general, any method used to calculate the Hurst exponent $H$ (see Sec. 7.1) can be adapted to obtain the generalized exponents $H_{q}$. For example, the multifractal generalization of the DFA consists in calculating the $q$ th-order fluctuation function $F_{q}(\tau)$,

$$
F_{q}(\tau)=\left\{\frac{1}{N \tau} \sum_{t=1}^{N \tau}\left|X(t)-Y_{\tau}(t)\right|^{2 q}\right\}^{1 / 2 q}
$$

In complete analogy with (148), the exponents $H_{q}$ are then obtained from the scaling

$$
F_{q}(\tau)=C_{q} \tau^{H_{q}}
$$

[We remark parenthetically that the multifractal DFA defined above is slightly different from the formulation introduced in Ref. [46], but such minor distinctions do not matter in practice.]

As an illustration of the multifractal DFA, we have applied this method to the Ibovespa returns. For each $q$ we computed the function $F_{q}(\tau)$ defined in (164), plotted it in a double-logarithmic scale, and obtained the generalized exponent $H_{q}$ from the slope of the curve. In Fig. 12 it is plotted the resulting quantity $q H_{q}$ as a function of $q$. In this figure we clearly see that $q H_{q}$ deviates from the linear behavior expected for a monofractal, thus indicating that the time series of Ibovespa returns does indeed display multifractal behavior. Evidences of multifractal behavior have been seen in several other stock indexes [41].

Multifractality has also been observed in many time series for currency exchange rates, and this has motivated the suggestion that there might perhaps be a formal analogy between turbulent flows and foreign currency exchange markets; see Ref. [11] for references to the original literature. In turbulence [47], there is an energy cascade from the large scales (where energy is fed into the system) down to the small scales (where energy is dissipated by viscosity). In currency markets the analog would be a kind of information cascade in time from long-term investors (that lake a longer view of the market) to short-term investors (that watch the market very frequently) [11]. 


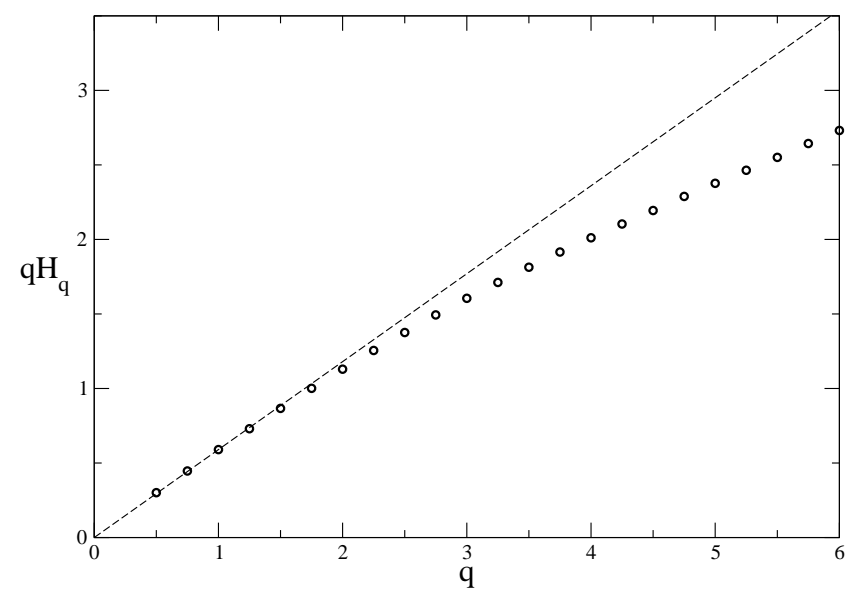

Figure 12. The generalized Hurst exponents $H_{q}$ as a function of $q$ for the Ibovespa. The dashed line indicates the linear behavior of a monofractal FBM with $H=0.6$.

\section{Conclusions}

In these notes, I tried to present a basic introduction to an interdisciplinary area that has become known, at least among physicists working on the field, as Econophysics. I started out by giving some basic notions about financial derivatives and illustrated how to price them with a simple binomial model. After this motivational introduction, I offered a concise description of Brownian motion and stochastic calculus, which provide the necessary mathematical tools to describe financial asset prices in continuous time. I then proceeded to discuss the Standard Model of Finance (SMF), namely, the Black-Scholes model for pricing financial derivatives. The formulation of the Efficient Market Hypothesis, which lies at the heart of the SMF, in terms of martingales and its consequences for pricing derivatives were also discussed. Finally, I briefly reviewed some recent work done mostly, but not exclusively, by physicists that have produced evidences that the SMF may not fully describe real markets. In this context, some possible extensions of the Black-Scholes model were considered.

I should like to conclude by mentioning that other alternatives approaches to the problem of pricing financial derivatives have been proposed by physicists, using methods originally developed to treat physical problems. For instance, the option pricing problem was recently discussed in the context of the so-called non-extensive statistical mechanics [48]. A "Hamiltonian formulation" for this problem was also given in which the resulting "generalized BlackScholes" equation is formally solved in terms of path integrals [49]. We refer the reader to Ref. [11] for a brief review of these and other recent developments in Econophysics.

\section{Acknowledgments}

I am grateful to Rogério L. Costa for many useful discussions and for providing Fig. 12. Financial support from the Brazilian agencies CNPq and FINEP and from the special research programs PRONEX is acknowledged.

\section{A Some Basic Definitions from Prob- ability Theory}

\section{A.1 Probability space}

In probability theory one usually imagines performing an experiment in which chance intervenes. The occurrence or nonoccurrence of such an experiment is called an outcome $\omega$. The set of all possible elementary outcomes is denoted by $\Omega$.

An event $A$ is a set of outcomes, i.e., a subset of $\Omega$. We are interested in attributing a probability to events in $\Omega$. If $\Omega$ is finite or countable, we could introduce a probability $P(\omega)$ for each individual outcome $\omega$ and then define the probability $P(A)$ of an event $A$ as the sum of the probabilities of all outcomes that make up the event $A$ :

$$
P(A)=\sum_{\omega \in A} P(\omega) .
$$

This procedure, however, will not work when $\Omega$ is uncountable, i.e., $\Omega$ is a continuous space such as $\mathbf{R}$, since in this case the probability of any particular outcome is zero. Furthermore, a typical event will have uncountably many (i.e. a continuum of) outcomes. Hence the formula above is not applicable. That's why we need the notion of a probability measure to be defined shortly.

To do this, first we need to specify the class of 'observable events', i.e., the subsets of $\Omega$ to which a probability can be associated. If $\Omega$ is finite or countable, a partition of $\Omega$ would be the natural candidate. (Recall that a partition $\left\{A_{i}\right\}$ of a set $\Omega$ is a collection of disjoint subsets, i.e., $A_{i} \subset \Omega$ and $A_{i} \cap A_{j}=\emptyset$ for $i \neq j$, whose union covers the whole set $\Omega$, i.e., $\bigcup_{i} A_{i}=\Omega$.) In the case of a continuous space this is not possible and a different class of subsets is in order. To be useful, such a class must be closed under the various set operations, such as union, intersection, complementarity, etc. This is done through the concept of a $\sigma$-algebra.

Definition 10 A family $\mathcal{F}$ of subsets of $\Omega$ is a $\sigma$-algebra on $\Omega$ if the following conditions are fulfilled:

1. $\emptyset \in \mathcal{F}$ and $\Omega \in \mathcal{F}$

2. $A \in \mathcal{F} \Longrightarrow A^{c} \in \mathcal{F}$, where $A^{c}=\Omega \backslash A$ is the complement of $A$ in $\Omega$

$$
\text { 3. } A_{1}, A_{2}, \ldots \in \mathcal{F} \Longrightarrow \bigcup_{i=1}^{\infty} A_{i} \in \mathcal{F}
$$

The par $(\Omega, \mathcal{F})$ is called a measurable space.

The elements $A \subset \mathcal{F}$ of the $\sigma$-algebra $\mathcal{F}$ are called measurable sets or simply events. The idea here is that for a given set $A \in \mathcal{F}$ it is possible to ascertain whether any outcome $\omega$ belongs or not to $A$, and in this sense the event $A$ is observable.

The smallest $\sigma$-algebra consists of the empty set $\emptyset$ and the set $\Omega$ itself, i.e., $\mathcal{F}_{\text {min }}=\{\emptyset, \Omega\}$. The largest $\sigma$-algebra, 
on the other hand, is made up of all subsets of $\Omega$, which is known as the power set of $\Omega$ and denoted by $2^{\Omega}$, hence $\mathcal{F}_{\text {max }}=2^{\Omega}$. Intermediate $\sigma$-algebras can be generated in the following way. Start with a given family $\mathcal{U}$ of subsets of $\Omega$ and form the intersection of all $\sigma$-algebras that contain $\mathcal{U}$ :

$$
\mathcal{F}_{\mathcal{U}}=\bigcap\{\mathcal{F} \mid \mathcal{F} \supset \mathcal{U}\}
$$

In other words, $\mathcal{F}_{\mathcal{U}}$ is the smallest algebra that contains $\mathcal{U}$ as is called the algebra generated by $\mathcal{U}$.

We can now attribute a 'probability of occurrence' to events $A \in \mathcal{F}$ via a probability measure on $(\Omega, \mathcal{F})$.

Definition 11 A probability measure $P$ on the measurable space $(\Omega, \mathcal{F})$ is a function $P: \mathcal{F} \rightarrow[0,1]$ such that

1. $P(\emptyset)=0$ and $P(\Omega)=1$

2. If $A_{1}, A_{2}, \ldots \in \mathcal{F}$ is a disjoint collection of elements of $\mathcal{F}$, i.e., $A_{i} U A_{j}=\emptyset$ if $i \neq j$, then $P\left(U_{i=1}^{\infty} A_{i}\right)=$ $\sum_{i=1}^{\infty} P\left(A_{i}\right)$

The triple $(\Omega, \mathcal{F}, P)$ is called a probability space.

\section{A.2 Random variables}

Intuitively, a random variable $X$ is a function that attributes to each outcome $\omega$ a real number $x$, i.e., $X(\omega)=x$. We usually think of $X$ as a random number whose value is determined by the outcome $\omega$. A more formal definition is given in terms of measurable functions.

Definition 12 Let $(\Omega, \mathcal{F}, P)$ be a probability space. A function $f: \Omega \rightarrow \mathbf{R}$ is measurable with respect to the $\sigma$-algebra $\mathcal{F}$, or more compactly, $\mathcal{F}$-measurable, if

$$
f^{-1}(U) \equiv\{\omega \in \Omega \mid f(\omega) \in U\} \in \mathcal{F},
$$

for all open sets $U \in \mathbf{R}$.

The definition above means that for any given interval $(a, b) \subset \mathbf{R}$ there is a meaningful event $A$ in $\Omega$. In an abuse of language we usually refer to this event as $A=\{a<f<$ $b\}$.

Definition 13 A random variable $X$ on a probability space $(\Omega, \mathcal{F}, P)$ is a $\mathcal{F}$-measurable function.

A random variable $X$ naturally generates a $\sigma$-algebra. This is the algebra generated by all the sets $X^{-1}(U), U \subset$ $\mathbf{R}$ open, and is denoted $\mathcal{F}_{X}$. We think of $\mathcal{F}_{X}$ as representing the 'information' generated by the random variable $X$. The $\sigma$-algebra $\mathcal{F}_{X}$ contains the essential information about the structure of the random variable $X$; it contains all sets of the form $\{\omega \mid a<X(\omega)<b\}$.

We also recall the definition of the probability distribution $F(x)$ of a random variable $X$ :

$$
F(x)=P(X \leq x), \quad \text { for } \quad x \in \mathbf{R} .
$$

Random variables can be either discrete, if the only assume a finite or countably number of values $x_{1}, x_{2}, \ldots$, or continuous. Most continuous distributions of interest have a density $f(x)$, i.e., a function $f(x)$ such that

$$
F(x)=\int_{-\infty}^{x} f(x) d x
$$

This allows us to compute the probability of a given event $A=\{a \leq X \leq b\}$ explicitly through the formula

$$
P(A)=\int_{a}^{b} f(x) d x .
$$

\section{A.3 Stochastic processes}

Intuitively, a stochastic process represents a dynamical system which evolve probabilistically in time. A formal definition is given below.

Definition 14 A stochastic process is a collection of random variables

$$
\left\{X_{t}\right\}_{t \in T}
$$

defined on some probability space $(\Omega, \mathcal{F}, P)$ and parametrized by the variable $t$.

We usually think of the label $t$ as being time, so that $X_{t}$ would represent the (random) value of the quantity $X$, say, the price of a risky asset or the position of a Brownian particle, at time $t$. For most of the cases, we consider $T$ to be the halfline $[0, \infty)$. In this case we have a continuous-time process. Eventually, we shall also consider discrete-time processes, in which case the variable $t$ assumes (non-negative) integer values, i.e., $T=\{0,1,2, \ldots\}$.

It is perhaps worth emphasizing that a stochastic process is a function of two variables. For a fixed time $t, X_{t}$ is a function of the random variable $\omega$, i.e., $X_{t}=X_{t}(\omega)$. For a fixed outcome $\omega \in \Omega$, it is a function of time, $X_{t}=X_{t}(\omega), \quad t \in T$. This function of time is called a realization, path, or trajectory of the stochastic process $X_{t}$. Note, in particular, that in this context an outcome corresponds an entire realization or trajectory of the stochastic process $X$.

A stochastic process is usually described in terms of the distributions it induces. The finite-dimensional distributions of the stochastic process $X_{t}$ are the joint probability distributions $p\left(x_{1}, t_{1} ; \ldots, x_{n}, t_{n}\right)$ of the random variables $X_{t_{1}}, \ldots, X_{t_{n}}$, for all possible choices of times $t_{1}, \ldots, t_{n} \in T$ and every $n \geq 1$. The finite-dimensional distributions determine many (but not all) relevant properties of a stochastic process. A special class of stochastic processes are the stationary ones.

Definition 15 A stochastic process is said to be stationary if all its finite-dimensional distributions are invariant under a time translation, that is,

$$
p\left(x_{1}, t_{1}+\tau ; \ldots, x_{n}, t_{n}+\tau\right)=p\left(x_{1}, t_{1} ; \ldots, x_{n}, t_{n}\right),
$$

for any $\tau>0$.

Another important class of stochastic process are Gaussian processes, where all finite-dimensional distributions are (multivariate) Gaussians. 


\section{References}

[1] J. C. Hull, Options, Futures, and Other Derivatives, 3rd ed., Prentice-Hall, Upper Saddle River, NJ, 1997.

[2] D. Duffie, Dynamic Asset Pricing, 3rd ed., Princeton University Press, Princeton, NJ, 2001.

[3] J. E. Ingersoll, Theory of Financial Decision Making, Rowman \& Littlefield, Savage, MD, 1987.

[4] T. Bjork, Arbitrage Theory in Continuous Time, Oxford Univ. Press, New York, 1998.

[5] A. N. Shiryaev, Essentials of Stochastic Finance: Facts, Models, Theory, World Scientific, Singapore, 1999.

[6] P. Wilmott, J. Dewynne, and S. Howison, The Mathematics of Financial Derivatives (A Student Introduction), Cambridge Univ. Press, Cambridge, 1996.

[7] T. Mikosch, Elementary Stochastic Calculus: with Finance in View, World Scientific, 1998.

[8] B. Øksendal, Stochastic Differential Equations: an Introduction with Applications, 5th ed., Springer, Berlin, 1998.

[9] R. Mantegna and H. E. Stanley, An Introduction to Econophysics, Cambridge Univ. Press, Cambridge, 2000.

[10] J.-P. Bouchaud and M. Potters, Theory of Financial Risks: From Statistical Physics to Risk Management, Cambridge Univ. Press, Cambridge, 2000.

[11] J. Voit, The Statistical Mechanics of Financial Markets, Springer, Berlin, 2003.

[12] F. Black and M. Scholes, J. Polit. Econ. 81, 637 (1973)

[13] R. C. Merton, Bell J. Econ. Manag. Sci. 4, 141 (1973)

[14] Bachelier, La Thóerie de la Spéculation [Ph.D. thesis in mathematics], Ann. Sci. Ecole Norm. Super., Sér. 3, 17, 21 (1900). Translated in: P. H. Cootner (ed.), The Random Character of Stock Market Prices, MIT Press, Cambridge, MA, 1988.

[15] F. Reif, Fundamentals of Statistical and Thermal Physics, McGraw-Hill, Tokyo, 1965.

[16] A. Einstein, Ann. Physik (Leipzig) 17, 549 (1905)

[17] N. Wiener, J. Math. Phys. Math. 2, 131 (1923).

[18] K. Itô, Proc. Japan Acad. 22, 32 (1946); Mem. Amer. Math. Soc. 4, 1 (1951).

[19] C. W. Gardiner, Handbook of Stochastic Methods: for Physics, Chemistry, and the Natural Sciences, 2nd ed., Springer, Berlin, 1985.

[20] A. G. Malliaris and W. A. Brock, Stochastic Methods in Economics and Finance, North-Holland, Amsterdam, 1982.

[21] P. Billingsley, Probability and Measure, 3rd ed., Wiley, New York, 1995.

[22] B. Mandelbrot, J. Business 40, 394 (1963).

[23] H. M. Gupta and J. R. Campanha, Physica A 268, 231 (1999).

[24] R. Matsushita, P. Rathie, S. da Silva, Physica A 326, 544 (2003).

[25] L. C. Miranda and R. Riera, Physica A 297, 509-520 (2001).
[26] W. Schoutens, Lévy Processes in Finance: Pricing Financial Derivatives, Wiley, 2003.

[27] A. Matacz, Int. J. Theor. Appl. Financ. 3, 143-160 (2000).

[28] J. L. McCauley and G. H. Gunaratne, Physica A 329, 178-198 (2003).

[29] A. C. Silva, R. E. Prange, and V. M. Yakovenko, condmat/0401225, 2004.

[30] K. Matia, M. Pal, H. E. Stanley, and H. Salunkay, Europhys. Lett. 66, 909-914 (2004).

[31] M. S. Taqqu, V. Teverovsky, W. Willinger, Fractals 3 (1995) 785.

[32] H. E. Hurst, Trans. Am. Soc. Civ. Eng. 116 (1951) 770; H. E. Hurst, R. P. Black, and Y. M. Simaika, Long-Term Storage: An Experimental Study, Constable, London, 1965.

[33] C.-K Peng, S. V. Buldyrev, S. Havlin, M. Simons, H. E. Stanley, A. L. Goldberger, Phys. Rev. E 49 (1994) 1685.

[34] J. G. Moreira, J. Kamphorst Leal da Silva, S. Oliffson Kamphorst, J. Phys. A 27 (1994) 8079.

[35] R. L. Costa and G. L. Vasconcelos, Physica A 329, 231 (2003).

[36] K. Hu, P. Ch. Ivanov, Z. Chen, P. Carpena, H. E. Stanley, Phys. Rev. E 64 (2001) 011114.

[37] B. Mandelbrot and J. W. van Ness, SIAM Review 10, 422437 (1968)

[38] E. E. Peters, Fractal Market Analysis: Applying Chaos Theory to Investment and Economics, Wiley, New York, 1994.

[39] N. Vandewalle, M. Ausloos, Physica A 246 (1997) 454.

[40] P. Grau-Carles, Physica A 287 (2000) 396.

[41] T. Di Matteo, T. Aste, M. M. Dacorogna, Physica A 324, 183 (2003); Cond-Mat preprint 0302434, 2003.

[42] Y. Hu and B. Øksendal, "Fractional white noise calculus and applications to Finance," Preprint University of Oslo (2000).

[43] C. Necula, "Option pricing in a fractional Brownian motion environment," Preprint Academy of Economic Studies, Bucharest (Romenia), 2002.

[44] D. O. Cajueiro and J. J. Barbachan, "Volatility estimation and option pricing with the fractional Brownian motion," preprint, 2003.

[45] E. Ott, Chaos in Dynamical Systems, Cambridge University Press, Cambridge, 1993.

[46] J. W. Kantelhardt, S. A. Zschiegner, E. Koscielny-Bunde, A. Bunde, S. Havlin, and H. E. Stanley, Phys. Rev. E (2001).

[47] L. Landau and E.M. Lipschitz, Fluid Mechanics, Pergamon Press, Oxford, 1987.

[48] R. Osorio, L. Borland, and C. Tsallis, in Nonextensive Entropy: Interdisciplinary Applications, C. Tsallis and M. Gell-Mann (eds.), Oxford Press, 2004; L. Borland, Phys. Rev. Lett. 89, 098701 (2002).

[49] H. Kleinert, Physica A 312, 217 (2002). 